\title{
DEVELOPMENT OF A THICK GAS ELECTRON MULTIPLIER BASED BETA-RAY DETECTOR
}




\title{
DEVELOPMENT OF A THICK GAS ELECTRON MULTIPLIER BASED BETA-RAY DETECTOR
}

BY:

MATTHEW R. BERNACCI, B.SC.

\begin{abstract}
A Thesis Submitted to the School of Graduate Studies for Fulfilment of the Requirements for the Degree

Master of Science
\end{abstract}

McMaster University

(C) Copyright by Matthew Bernacci, 2018 
MASTER OF SCIENCE (2018)

(Radiation Sciences Graduate Program)
McMaster University

Hamilton, Ontario
TITLE:

AUTHOR:

SUPERVISOR:

NUMBER OF PAGES: $\quad \mathrm{x}, 60$

Development of a Thick Gas Electron Multiplier Based Beta-ray

Detector

Matthew R. Bernacci

B.Sc., (Medical Physics Co-op)

McMaster University, Hamilton, Canada

Dr. Soo-Hyun Byun 


\begin{abstract}
A new beta-ray detector using the Thick Gas Electron Multiplier (THGEM) technology is presented. Traditional proportional counters have been considered the standard for many decades for radiation contamination monitoring. However, it has always been challenging to detect low energy beta-emitters such as ${ }^{3} \mathrm{H}$ and ${ }^{14} \mathrm{C}$. In order to extend the low energy cut-off of these beta particles, it is important to keep the electron multiplication gain as high as possible. To accomplish this goal, we have developed a new gaseous beta-ray detector using THGEMs. Founded on previous THGEM avalanche simulations [1] and predecessor detectors, a novel prototype THGEM beta-ray detector was designed and fabricated. Its signal performance, effective gain and gain stability were comprehensively studied for single and double-THGEM configurations using an alpha source. The first time THGEM detector response to beta-rays was observed for various operating conditions and compared with Monte Carlo N-Particle Transport 6 (MCNP6) Monte Carlo simulations.
\end{abstract}




\section{Acknowledgments}

Firstly, I would like to say how truly grateful I am for working under the supervision of Dr. Soo-Hyun Byun. As a great mentor, you have helped me considerably enhance my skills, knowledge, and ability to overcome new challenges. Even as my request to discuss a problem for a few minutes usually evolved into an hour-long discussion, you wouldn't dismiss me until I was headed in the right direction. I also thank you for giving me a unique, rewarding yet challenging project with plenty of opportunity to learn new skills. I would also like to give a special thanks to my committee members Dr. David Chettle, and Dr. Alan Chen. Your assistance on statistics, and important ideas to consider during my project were vital throughout my M.Sc. research.

With respect to designing my detector and the related electronics, I have several people who I am sincerely thankful for. Most importantly, Dr. Andrei Hanu, whose innovative previous work with THGEMs provided huge inspiration and a strong foundation when designing this detector. In addition, Eric Johnston, machinist Jim Cleaver and Justin Bennet, as well as NEUDOSE members who advised and helped fabricate many of the necessary components. To the Health Physics Department at McMaster, particularly Chris Malcolmson, Derek Cappon, Glenn McClung and all the rest. Thank you for providing us with valuable research tools including radiation sources, and the use of a windowless proportional counter, in addition to reputably safe radioactive work environments. 
I would like to sincerely thank Duaa Alaroui, for being a great travel companion, research assistant, and academic editor. Your positivity and sense of humour helped me stay motivated throughout my project. To the rest of our members in our expanding research group, particularly Devin Burke, Ye Eun, and Xin Tong, thank you for supporting me as I developed my detector. Also to the primary occupants of TAB 104C, and housemates of 31 Thorndale, thank you for providing me with laughter, moral support, and an educational space to share over the years.

Lastly, I would like to thank my mother Carol, father Rob, sister Katelyn and new brother-in-law Kris, my old dog and study buddy Tara, in addition to the rest of my family including my Oma, Nonno, and finally grandpa Thom and grandma Monica, who have been looking down upon me during this incredible experience. Without my mother's infinite patience, and unparalleled support this would not have been made possible. I can't express how vital you are to our family. To my dad, who has constantly challenged me to do better and immense work ethic as an entrepreneur inspired me to achieve my goals. 


\section{Contents}

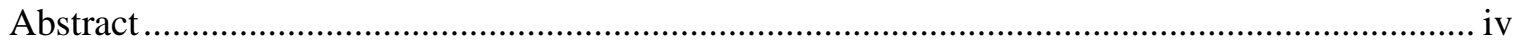

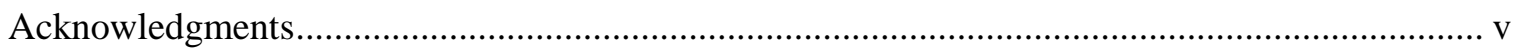

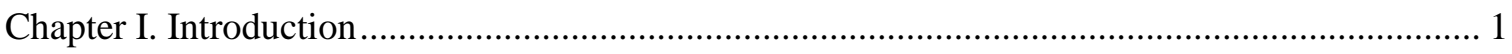

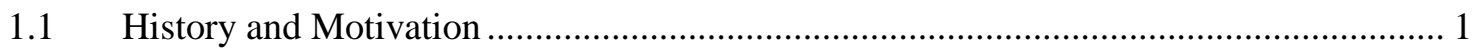

1.2 Beta-ray Contamination Sources.......................................................................... 5

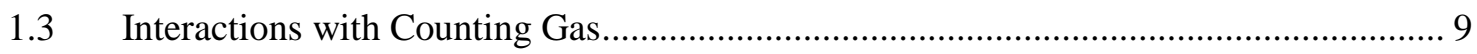

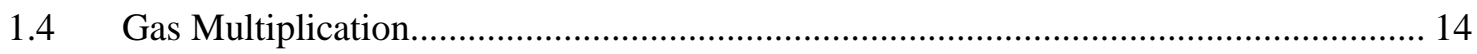

1.5 Traditional Proportional Counter and THGEM Comparison....................................... 15

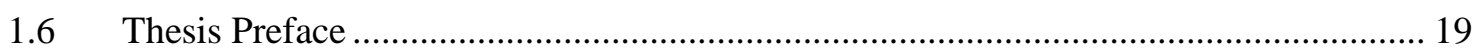

Chapter II. THGEM Beta-ray Detector Design and Pulse Processing System ............................ 20

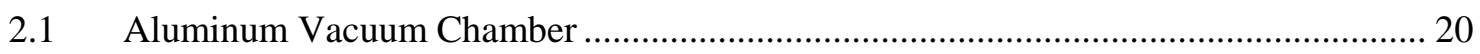

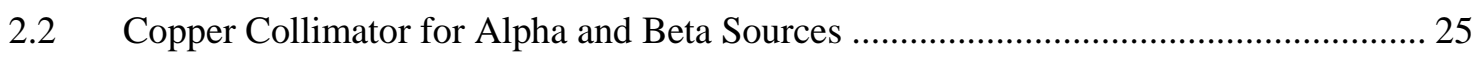

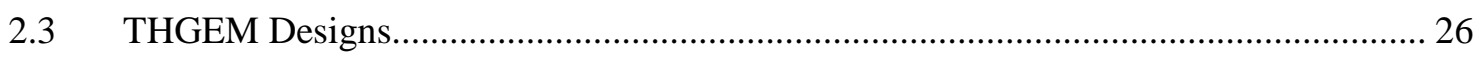

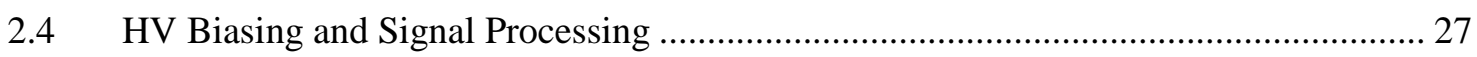

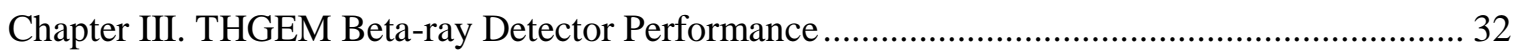

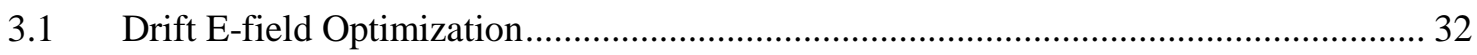

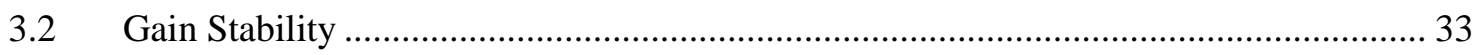

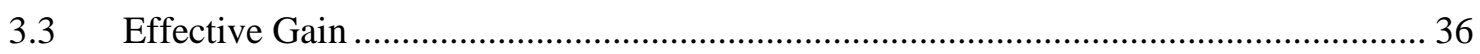

3.4 ${ }^{14} \mathrm{C}$ Disk Source Beta-ray Spectra and Counting Curves ........................................... 42

3.5 ${ }^{14} \mathrm{C}$ Energy Calibration and MCNP6 Pulse Height Spectra Comparison ....................... 45

3.6 ${ }^{14} \mathrm{C}$ Disk Source Beta-ray Stability Measurements ................................................ 47

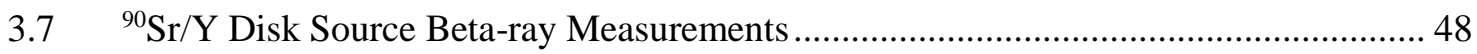

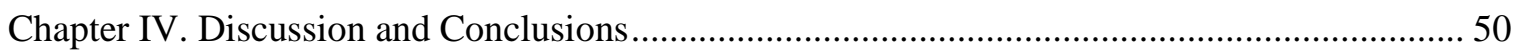

4.1 Insulator Charge Build-up and Ion Back-flow (IBF) Effect on Gain............................. 50

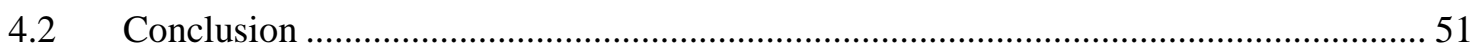

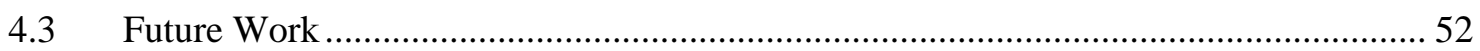

Appendix A.1: MCNP6 Deck for ${ }^{244} \mathrm{Cm}$ Alpha Source with Collimator ....................................... 56

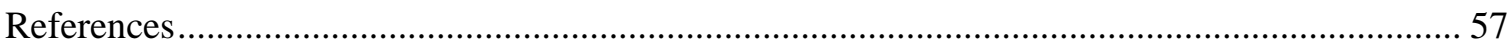




\section{List of Figures}

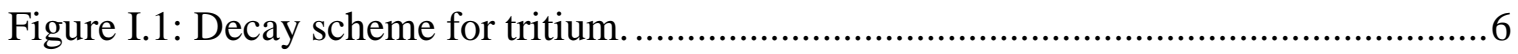

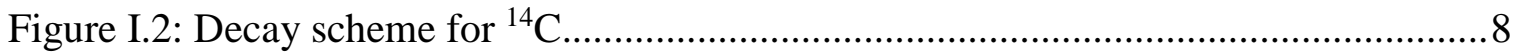

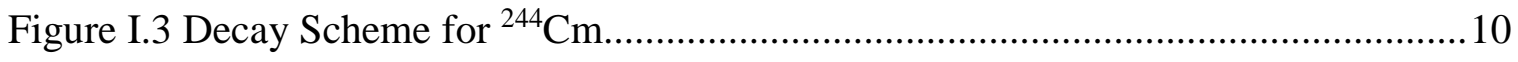

Figure I.4: Stopping Power for electrons in propane-based tissue equivalent (TE) gas, a common counting gas used in proportional counters [31] ........................................13

Figure I.5: A simplified illustration of a traditional proportional counter. .......................16

Figure I.6: Overall structure of THGEM assembly with typical spacing dimensions.......17

Figure I.7: Functional regions of a THGEM assembly with dimensions of utilized

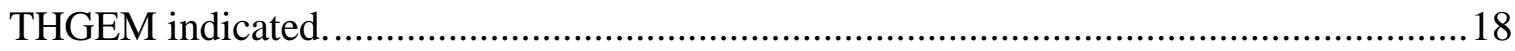

Figure II.1: Technical drawings for base of aluminum vacuum chamber. ......................21

Figure II.2: Technical drawings for lid of aluminum vacuum chamber. ........................22

Figure II.3: 3D printed SHV insulators used to prevent discharge between connector and

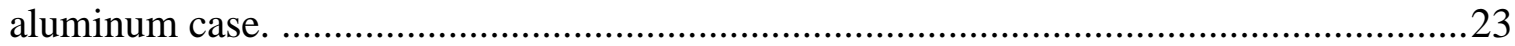

Figure II.4: Aluminum vacuum chamber base and lid with double-THGEM and

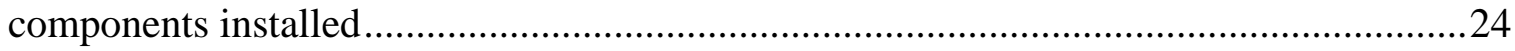

Figure II.5: Alpha (left) and beta (right) copper source holders...................................25

Figure II.6: THGEMs fabricated by Milplex Circuit (Canada) Inc, and Readout Board PCBs fabricated by OSH Park. THGEM 3 can interface well to increase active area as

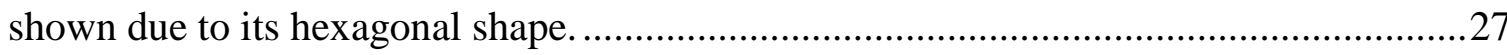

Figure II.7: Image of burnt THGEM hole from electrical breakdown due to a non-uniform copper surface.

Figure II.8: Example of older voltage divider (left) and newer model (right) with HV noise filtering. The newer model (still under development) will be able to provide noise filtering and bias double-THGEMs and the cathode with one PCB, whereas the older version required two identical PCB dividers in series to bias double-THGEMs..............28

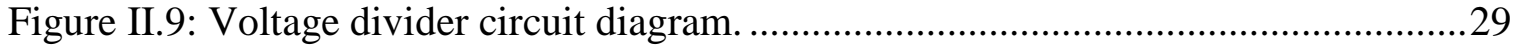

Figure II.10: Block diagram with signal processing equipment used............................31

Figure II.11: Lid of aluminum chamber with two high vacuum SHV connectors and one high vacuum BNC connector for signal output to preamplifier. .31 
Figure II.12: Experimental layout showing most pulse processing equipment in NIM bins.

Figure III.1: Drift E-field optimization using ${ }^{244} \mathrm{Cm}$ with a $1 \mathrm{~cm}$ SV height.....................33

Figure III.2: Experimental and simulated ${ }^{244} \mathrm{Cm}$ spectrum, both fit with exponentially modified Gaussian function with $95 \%$ confidence bounds. Experimental spectra measured at 100 torr TE-propane gas using single-THGEM.....

Figure III.3: MCNP6 detector with source geometry in green for $2 \times 1 \mathrm{~mm}$ collimator. 5 $\mathrm{mm}$ diameter disk source with isotropic emission was modelled.

Figure III.4: Long term ${ }^{244} \mathrm{Cm}$ stability for single-THGEM in 100 torr TE-propane gas..36 Figure III.5: A set of ${ }^{244} \mathrm{Cm}$ spectra for single- and double-THGEM configurations at 100 torr TE-propane.

Figure III.6: Pulser calibration with two sets of measurements taken approximately 1 month apart to check for reproducibility, for amplifier gain of 90× using ORTEC 109a test input

Figure III.7: Effective gain curves for 100 torr TE-propane and P10 using ${ }^{244} \mathrm{Cm}$. Transfer region and collection gap were increased from 3 to $4.5 \mathrm{~mm}$ and 1.5 to $3 \mathrm{~mm}$ respectively to increase maximum operating voltage before sparking was induced.

Figure III.8: Preamplifier (200 mV/div) and shaping amplifier (5.00 V/div) oscilloscope

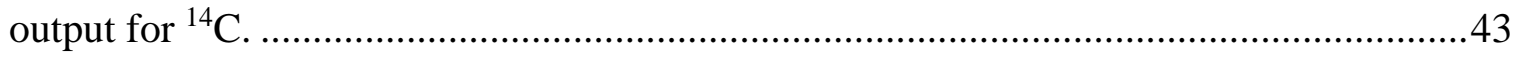

Figure III.9: Set of ${ }^{14} \mathrm{C}$ spectra for HV run for single-THGEM 100 torr TE-propane.......43

Figure III.10: Counting curves for 100 torr TE-propane and $\mathrm{P} 10$ gas using ${ }^{14} \mathrm{C} \ldots \ldots \ldots \ldots . . . .44$

Figure III.11: Experimental data for double THGEM in 100 torr TE-propane at $\Delta \mathrm{V}_{\text {THGEM }}=563 \mathrm{~V}$ with MCNP6 simulated spectra for comparison. One noticeable feature is the rise in counts in the last few channels, attributed to particles that were binned in

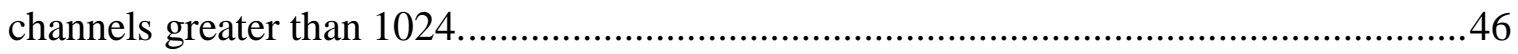

Figure III.12: MCNP6 simulation for ${ }^{14} \mathrm{C}$ in THGEM beta-ray detector at different gas pressures.

Figure III.13: ${ }^{14} \mathrm{C}$ source stability using net count rate (cps) at $\mathrm{HV}_{\text {cathode }}=1600$

$\mathrm{HV}_{\text {THGEM}}=1450 \mathrm{~V}, 100$ torr TE-propane gas.

Figure III.14: Set of spectra used to measure stability for double-THGEM at $\mathrm{HV}_{\text {cathode }}=$ $1500 \mathrm{~V}, \mathrm{HV}_{\text {THGEM }}=1350 \mathrm{~V}$ in 100 torr TE-propane gas.

Figure III.15: ${ }^{90} \mathrm{Sr} / \mathrm{Y}$ source stability with $200 \mathrm{~V}$ increments for $\mathrm{HV}_{\text {cathode }}$ in 100 torr TEpropane.

Figure IV.1: Three hexagonal THGEMs used to increase surface area.... 


\section{List of Tables}

Table II.1: THGEMs tested in order of increasing SV for $1 \mathrm{~cm}$ SV height......................26

Table II.2: Determination of pulse processing electronics with lowest noise levels.........30

Table III.1: Properties for two fill gases used for $\mathrm{E}_{\alpha}=5.794 \mathrm{MeV}$................................39

Table III.2: Comparison of first Townsend coefficient for two gases at low pressure and

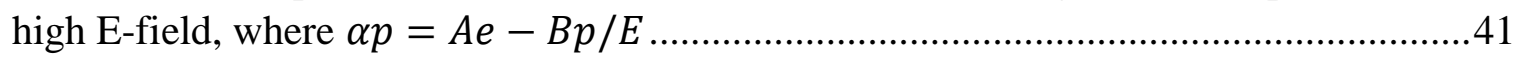




\section{Chapter I. Introduction}

\subsection{History and Motivation}

Gas-filled proportional counters are a group of radiation detectors crucial for many applications including contamination monitoring at nuclear facilities. Large area, cost effective detectors with high efficiencies, energy discrimination, and robust design are preferred for contamination control in these environments. The first counting tube was developed in 1908 by Rutherford and Hans Geiger [2], and was able to detect alpha particles penetrating a thin mica window installed on a sealed, low pressure hollow brass cylinder with a thin $0.45 \mathrm{~mm}$ diameter wire along its central axis. As the alpha particle traversed the cylinder it ionized gas within the chamber producing ion pairs (positive ions and electrons), which were accelerated by an applied E-field. The thin wire would serve as a collecting electrode, and a pulse would be produced manifesting as a sharp movement on the needle of an electrometer. An improvement was made in 1928 by Hans Geiger and Otto Klemperer, and the first proportional counter was conceived [3]. Based on the Geiger point counter, where a sharp point was used to produce an intense localized E-field, a small sphere was placed around the end of a central electrode, and the polarity was set such that the central electrode functioned as the anode. Low voltages allowed for the detection of alpha particles only, but the separate detection of alpha and beta particles was permitted when the applied voltage was increased beyond the critical voltage. This design served as the basis of proportional counters, given by their ability to differentiate 
particles by pulse height. Observed dead times were significantly shorter than GM detectors due to the avalanches in the chamber, created when electrons are accelerated at sufficient velocities in a gas, being isolated to a small region of the anode. In 1943, John Simpson invented the first gas-flow proportional counter using methane as counting gas with the purpose of measuring plutonium alpha-emitters in combination with betaradiation originating from fission products. The windowless chamber enabled insertion of the sample into the counter volume through a special gas lock. A short time constant reduced pulse pile up and provided increased rejection of the beta pulses [4]. Later he pioneered the use of P10 gas (10\% methane and 90\% argon), the most commonly found gas in proportional counters due to its low cost and low electron attachment coefficient $[5]$.

In 1968, a revolutionary multi-wired proportional chamber (MWPC) was successfully developed and operated by Charpak et al. [6]. Using the time delays of the pulses generated by particle tracks adjacent to the anode wires, it was possible to measure their positions with high precision. The detector was capable of high counting rates $\left(10^{5}\right.$ cps/wire), with 1-D position resolution of $100 \mu \mathrm{m}$ or better. It soon became the dominant detector in particle physics for the next few decades. Nevertheless, limitations include electrostatic repulsion from the close proximity of wires, wire tension stability, and Efield deterioration due to the space charge effect [7].

With advances in microelectronics, a new generation of gas filled detectors called MicroPattern Gas Detectors (MPGDs) was developed in the 1990s. The more robust electrodes were fabricated using newer technology, such as photolithography which 
enabled very fine structural detail and better spatial resolution than the MWPC. Although there are several designs of MPGDs existing today such as strip-type, dot-type, and parallel plate-type, the most popular type today is the hole-type [8]. In 1997, F. Sauli introduced a hole-type MPGD where a thin insulator material is coated on both sides with a metallic surface and etched with several through-holes [9]. Termed the Gas Electron Multiplier (GEM), it was situated between a drift and charge collection electrode which functioned to guide electrons through the holes and then onto the collection anode. When a large potential difference was applied across the metal layers of the GEM electrode, an intense E-field was created in each of the holes where gas multiplication could occur if the voltage surpassed a threshold. Distinct advantages over previous proportional chambers were the ability to insert multiple GEM assemblies to achieve higher gain, and the separation of the collection anode from the multiplication region to prevent positive ions from contributing to the signal output [10].

In 2005, Surette and Dubeau [11] developed a double-GEM detector capable of accurately measuring airborne tritium concentrations. Due to the common presence of other isotopes in sampled gases the amount of tritium measured is severely overestimated. In order to assess the hazard level of tritiated air, the detector was able to discriminate tritium from radiation originating from other sources using spatial ionization cluster size information. A readout array consisting of $11 \times 11$ square pads measuring $4 \times 4 \mathrm{~mm}$ was employed to determine the number of pads triggered by an ionizing particle. Due to the short range of tritium beta-rays, only one or two pads would trigger compared to a much larger number by other isotopes such as ${ }^{14} \mathrm{C}$. The double-GEM fixed tritium monitor 
displayed promising results and was successful in reducing spillover in the tritium channel to the order of $\pm 5 \%$ for equal concentrations of ${ }^{14} \mathrm{C},{ }^{85} \mathrm{Kr}$, or ${ }^{133} \mathrm{Xe}$ mixed with tritium.

One of the most recent developments based on this is the Thick Gas Electron Multiplier (THGEM), with a similar structure as the GEM but dimensions 5-20 times greater. This also allows it to be conveniently manufactured using commercial PCB design software and production facilities, compared to GEMs which require more specialized techniques to produce. THGEMs have an extensive list of applications and recent work with THGEMs include x-ray imaging [12], mixed neutron/gamma field microdosimetry [13,14], contraband detection using CsI coated THGEMs [15], and dark matter detection $[16,17]$. Due to the THGEM's simple, robust, and low cost per unit area properties it is an attractive detector for contamination monitoring of beta-ray emitting isotopes in nuclear facilities.

The presence of radioactivity can be detected using hand held survey instruments or permanent area monitors such as gaseous wire-anode proportional counters, which have been considered the standard device for many decades. Since controlled areas where contamination can be present can be quite large, detectors with increased surface areas are preferred for effectively monitoring these areas. For contamination monitoring upon exit of the on campus research reactor and high level radiation labs, McMaster University employs rectangular parallel plate proportional counters in their whole body monitors and hand held contamination friskers manufactured by Mirion Technologies. The whole body surface contamination monitor can detect beta-ray contamination on 
hands, shoes, and clothing. It consists of up to 25 detectors each $32.3 \times 11.6 \mathrm{~cm}^{2}$ in area, with 2 anode wires running through the centre of each at an operating voltage of $1650 \mathrm{~V}$. A thin titanium window guarded by a fine mesh for protection is used to contain the gas. Efficiency varies on contact with the outer mesh from $8 \%$ for low energy beta-emitters such as ${ }^{14} \mathrm{C}$ up to $32 \%$ for high energy ${ }^{90} \mathrm{Sr} /{ }^{90} \mathrm{Y}$ beta-rays[18]. Limitations of these commercial detectors include their reduced efficiency for low energy beta-rays, size, fragile structure, and limited surface area.

Thus, the two goals of this thesis are to firstly decrease the low energy cut-off of beta-ray spectra by increasing electron multiplication, and secondly investigate improvements for large area monitoring capabilities of proportional counters for betacontamination monitoring. To accomplish these goals, THGEM technology will be implemented. Once completed, this detector will help serve as a foundation for building a portable THGEM detector for monitoring larger surface areas and measuring sources of airborne tritium contamination.

\subsection{Beta-ray Contamination Sources}

When designing an instrument for detecting radiation, it is necessary to identify important isotopes and understand the type of contamination (fixed, loose, airborne) that may be present for its application. Although radioactive materials are intended to be contained, it is often spread as a result of equipment failure, chronic leaks, human error, and maintenance work. It is therefore important to detect contamination to account for 
internal and external exposures. This subchapter will consider two main isotopes present in CANDU and other nuclear power plants, which is one of the primary areas of application for this detector.

In CANDU reactor power stations, where heavy water $\left({ }^{2} \mathrm{H}_{2} \mathrm{O}\right.$, or $\left.\mathrm{D}_{2} \mathrm{O}\right)$ is used as the primary heat transport and moderator, tritium $\left({ }^{3} \mathrm{H}\right.$, or $\left.\mathrm{T}\right)$ has been historically responsible for $30-40 \%$ of the radiation dose received by personnel [19]. In Canada, there are currently 18 operating CANDU Reactors producing significant amounts of tritium. Tritium is an isotope of hydrogen with 1 proton and 2 neutrons, and exists everywhere that water is present. The half-life of tritium is 12.3 years, and it emits a continuous energy distribution of beta particles with an average and maximum kinetic energy of 5.7 $\mathrm{keV}, 18.6 \mathrm{keV}$ respectively (see Figure I.1).

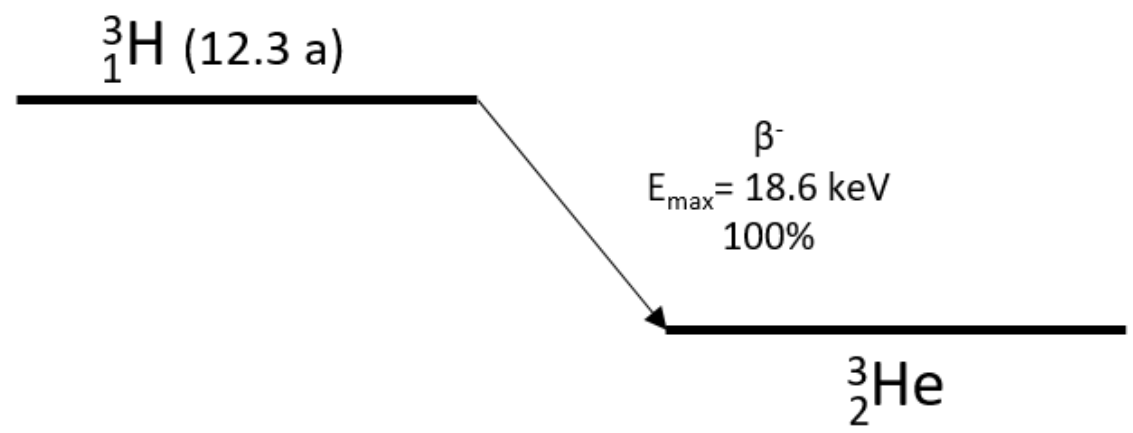

Figure I.1: Decay scheme for tritium.

Although tritium beta-rays only travel $6 \mathrm{~mm}$ in air and lack sufficient energy to penetrate the dead layer of skin, it poses a radiation hazard upon intake to the human body [20]. Tritium is produced when the deuterium atom $\left({ }^{2} \mathrm{H}\right.$, or D) in heavy water is exposed to neutrons through the following ${ }^{2} \mathrm{H}(\mathrm{n}, \gamma)^{3} \mathrm{H}$ reaction: 


$$
n+{ }_{1}^{2} H \rightarrow{ }_{1}^{3} H+\gamma
$$

Since tritium has similar chemical properties as that of ordinary hydrogen $\left({ }^{1} \mathrm{H}\right)$, anytime tritiated heavy water (TDO) exists and is exposed to air it will evaporate becoming an airborne tritium hazard. The majority of intake is due to inhalation and skin absorption, and is equally distributed among all body fluids via blood resulting in wholebody dose. At the Point Lepreau Nuclear Generating Station in 2001, tritium concentrations in the moderator heavy water indicated that air saturated at $35^{\circ} \mathrm{C}$ could expose unprotected workers in moderator system areas to doses of 3,000 mSv/h.

Additionally, tritium is encountered in areas involving the primary heat transport system such as the boiler room and fuelling machine vault, due to the high temperature and pressure causing leaks [19].

Carbon-14 $\left({ }^{14} \mathrm{C}\right)$ is another beta-emitting isotope of great interest. In 1985, during retubing of two CANDU reactors at Pickering Nuclear Generating Station, this previously undetected radioisotope was discovered throughout the station and even in employee's homes. As displayed in Figure I.2, ${ }^{14} \mathrm{C}$ has a half-life of 5730 years, with an average and maximum beta-ray energy of $49 \mathrm{keV}$ and $156 \mathrm{keV}$ respectively [21]. 


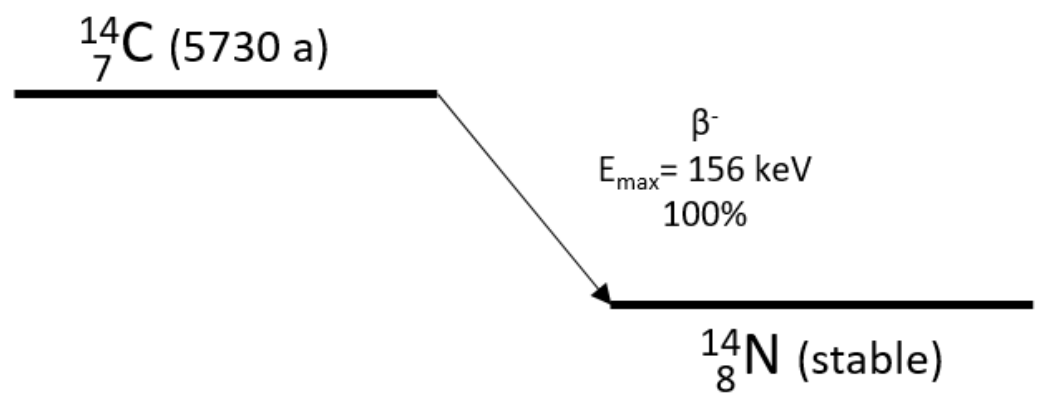

Figure I.2: Decay scheme for ${ }^{14} \mathrm{C}$.

It is produced through various neutron capture reactions, such as ${ }^{17} \mathrm{O}(\mathrm{n}, \alpha){ }^{14} \mathrm{C}$ and an ${ }^{14} \mathrm{~N}(\mathrm{n}, \mathrm{p}){ }^{14} \mathrm{C}$ reactions as follows [22]:

$$
\begin{aligned}
& { }_{8}^{17} O+n \rightarrow{ }_{6}^{14} C+\alpha \\
& { }_{7}^{14} N+n \rightarrow{ }_{6}^{14} C+p
\end{aligned}
$$

In CANDU reactors, estimates show $94.8 \%$ of ${ }^{14} \mathrm{C}$ is produced by neutron capture of ${ }^{17} \mathrm{O}$ present in $\mathrm{D}_{2} \mathrm{O}$ moderator which is exposed to high thermal neutron fluxes due to the higher isotopic abundance and inventory of ${ }^{17} \mathrm{O}$ [23]. Other main streams that contribute to the ${ }^{14} \mathrm{C}$ inventory in CANDU reactors are ${ }^{14} \mathrm{~N}$ and ${ }^{17} \mathrm{O}$ existing in dissolved air in the moderator system, ${ }^{17} \mathrm{O}$ in the primary heat transport system $\mathrm{D}_{2} \mathrm{O}$ (which is exposed to a smaller fraction of lower-flux thermal neutrons than $\mathrm{D}_{2} \mathrm{O}$ moderator), and $\mathrm{O}^{17}$ and $\mathrm{N}^{14}$ existing in $\mathrm{CO}_{2}$ and $\mathrm{N}_{2}$-based annulus gases respectively. It also builds up slowly in systems contaminated with air [23]. Similar to tritium, significant dose contribution from ${ }^{14} \mathrm{C}$ is through ingestion and inhalation pathways only. ${ }^{14} \mathrm{C}$ retention time in the human body depends on its chemical form, with short retention for $\mathrm{CO}$ and 
$\mathrm{CO}_{2}$ and longer lasting dose for organic compounds. It has a relatively long physical half-life, and for these reasons contamination monitoring is of great importance from a radiation protection standpoint. Due to its low energy, only thin window detectors permit the detection of ${ }^{14} \mathrm{C}$. The ${ }^{14} \mathrm{C}$ contact efficiency $(2 \pi)$ varies for commercially available window gas-filled detectors from $5 \%$ to $30 \%$ [18,24,25]. Windowless detectors offer the distinct advantage of increased counting efficiency because there is no barrier formed by the window, and the air gap between the sample and active volume is reduced.

\subsection{Interactions with Counting Gas}

Two of the main radiation sources used for testing the performance of the detector were Curium-244 $\left({ }^{244} \mathrm{Cm}\right)$ and ${ }^{14} \mathrm{C}$. The ${ }^{244} \mathrm{Cm}$ source has a probability of emitting two different alpha particles of similar energy, with an average energy of $5.794 \mathrm{MeV}$, via decay to ${ }^{240} \mathrm{Pu}$ as shown in Figure I.3. This alpha source had an activity of $3.70 \mathrm{kBq}$ with a reference date of Aug 1, 2011. The source was mounted above the sensitive volume in a copper source holder that functioned also as a collimator and cathode. The second source used was ${ }^{14} \mathrm{C}$, which emits a continuous energy range of beta-rays with a maximum kinetic energy of $156 \mathrm{keV}$ as previously discussed. The beta-ray source was manufactured in Nov 1, 1967 with an estimated activity of $37.0 \mathrm{kBq}$. Similar to the

${ }^{244} \mathrm{Cm}$ source, it was mounted in a copper source holder situated above the sensitive volume of the detector so that source particles may deposit their energy in this region filled with a counting gas (this will be elaborated on in section 2.2). 


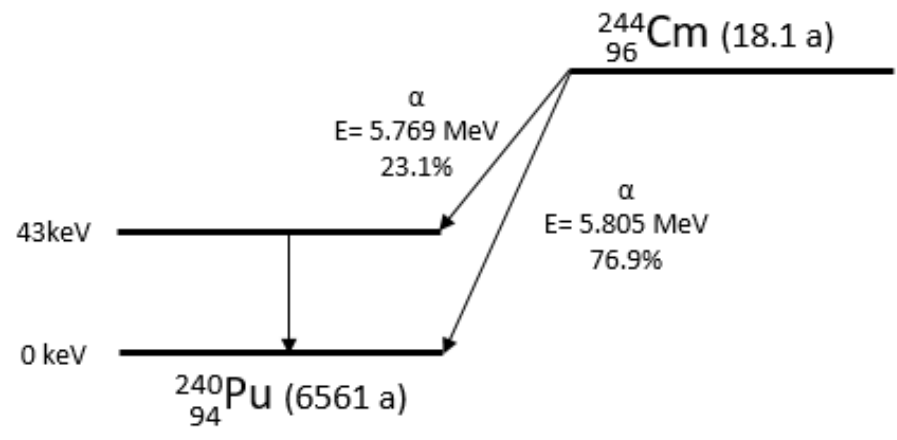

Figure I.3 Decay Scheme for ${ }^{244} \mathrm{Cm}$.

As a charged particle travels through the gas volume of a detector, it interacts and loses kinetic energy through collisional and radiative processes with the gas molecules. The probability of each process is given by their respective interaction cross sections. For particle energies involved in this thesis, radiative energy loss is negligible. Collision interactions for incident heavy charged particles involve inelastic collisions with atomic electrons, causing excitation or ionization. A charged particle typically interacts thousands of times in an object, and slows down until it is thermalized upon reaching velocities comparable to that of surrounding atoms in the medium. The linear energy lost per length in the gas volume is given by the linear stopping power. Bohr first derived a classical formula for calculating the linear stopping power for a particle. It assumes that electrons are free, in reality they are bound to atoms. Also it is not accurate for incident electrons because it assumes the incident particle is not deflected during collisions[26]. In addition, most radioactive beta-ray decays in the energy range of 1-10 MeV result in the emission of relativistic electrons which must be accounted for, although no alphas are considered relativistic from alpha decay [27]. This can be attributed to electrons having 
significantly lower mass than alpha particles, and requiring much less energy to accelerate them to relativistic velocities. H.A. Bethe provided the more exact quantum mechanical formula for linear stopping power pertaining to heavy charged particles as follows[28]:

$$
-\frac{d E}{d x}=\frac{4 \pi z^{2} e^{4} N Z}{m_{e} v^{2}}\left[\ln \frac{2 m_{e} v^{2}}{I}-\ln \left(1-\beta^{2}\right)-\beta^{2}\right]
$$

Where $\mathrm{Z}$ is the particle charge, $\mathrm{N}$ is the number density of target atoms, $\mathrm{Z}$ is the atomic number of target atoms, $\mathrm{v}$ is the velocity of the particle, $\beta=\mathrm{v} / \mathrm{c}$, and $\mathrm{I}$ is the mean excitation and ionization potential of the target medium. For non-relativistic charged particles such as alpha particles emitted by ${ }^{244} \mathrm{Cm}$, the last two terms cancel in the above equation. The Bethe formula is different for electrons (such as those emitted by ${ }^{14} \mathrm{C}$ ) because the electron has a significantly smaller mass than heavy particles, and there is a probability of the electron exchanging roles with the atomic electron, resulting in the atomic electron becoming the ionizing particle in the medium. The Bethe formula for electrons is given by [28]:

$$
\begin{aligned}
-\frac{d E}{d x}=\frac{2 \pi e^{4} N Z}{m_{e} v^{2}} & {\left[\ln \frac{m_{e} v^{2} E}{2 I^{2}\left(1-\beta^{2}\right)}-(\ln 2)\left(2 \sqrt{1-\beta^{2}}-1+\beta^{2}\right)+1-\beta^{2}\right.} \\
+ & \left.\frac{1}{8}\left(1-\sqrt{1-\beta^{2}}\right)^{2}\right]
\end{aligned}
$$


As briefly mentioned, heavily charged particles travel in a linear fashion which is not the case for electrons, which are subject to large angular changes upon interaction with target atoms. Additionally, at low electron energies, collision stopping power $\left(\mathrm{S}_{\mathrm{col}}\right)$ dominates where inelastic collisions with orbital electrons results in atomic ionization or excitation. At higher electron energies, radiative stopping power $\left(S_{\text {rad }}\right)$ surpasses $S_{\text {col }}$ due to the electron experiencing deceleration via coulomb interactions with the nucleus releasing a photon (called Bremsstrahlung) [29]. High energy beta-rays, resulting from fission product decay in nuclear reactors also emit radiative energy through Cherenkov radiation. An approximation for the ratio of $S_{\text {rad }}$ and $S_{\text {coll }}$ is given by [30]:

$$
\frac{S_{\text {rad }}}{S_{\text {coll }}} \approx \frac{2 E}{1600 m_{e} c^{2}}
$$

Consequently, it is assumed that most of the incident beta-rays and all primary electrons generated by incident particles involved in this thesis are low energy and lose most of their energy through collisions causing atomic ionization and excitation in the counting gas as shown by the low to mid energy range in Figure I.4. 


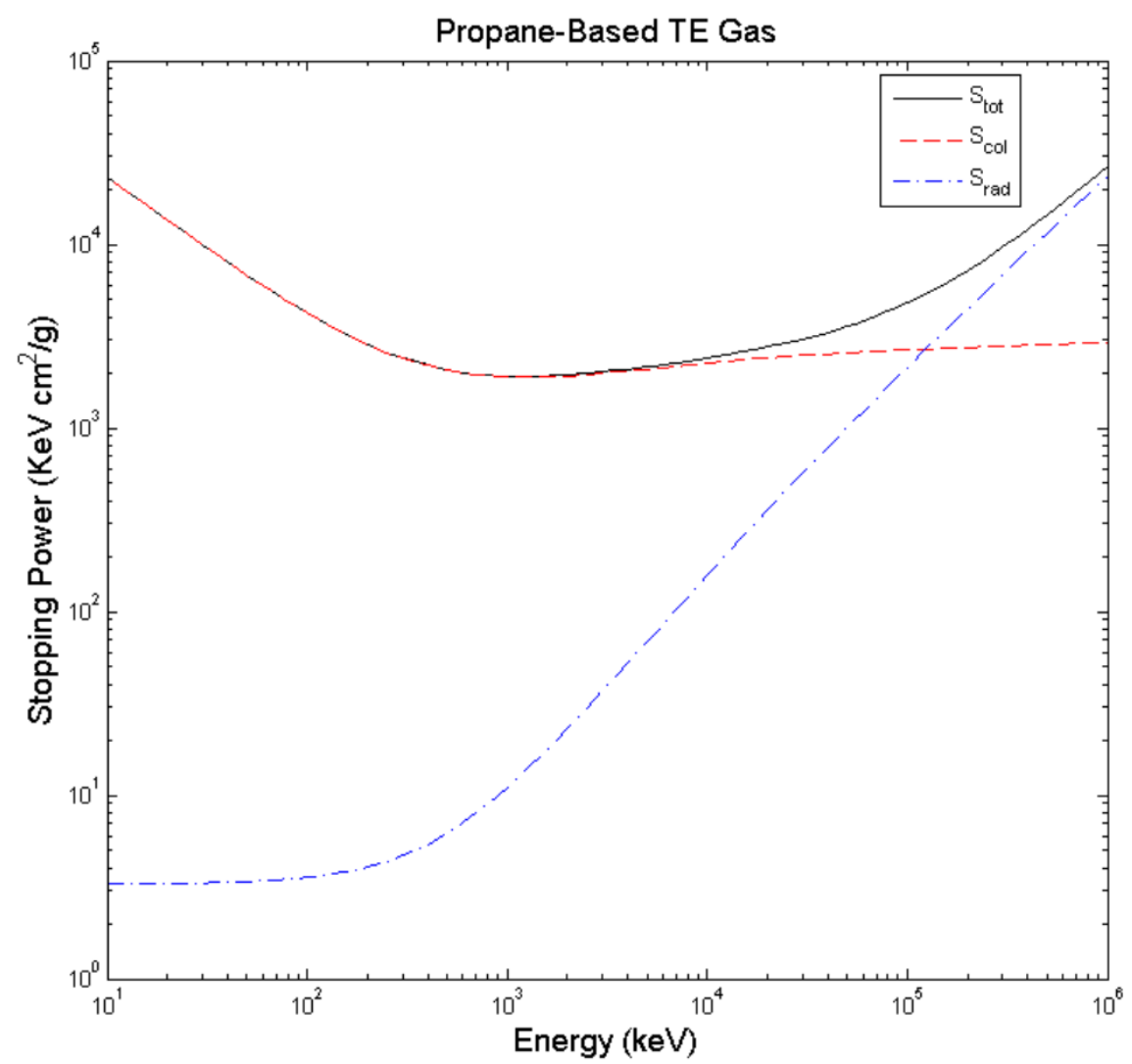

Figure I.4: Stopping Power for electrons in propane-based tissue equivalent (TE) gas, a common counting gas used in proportional counters [31].

In addition to the use of numerical methods for calculating the formation of ion pairs through collisions in a medium, an important quantity for estimating the mean number of ion pairs created along a particle track is called the $\mathrm{W}$-value. It is defined as the mean energy needed to produce an ion pair by a charged particle (eV/ion pair). Wvalue is dependent on the type of particle, particle energy, and medium it passes through. Utilizing a known $\mathrm{W}$-value, the average number of ion pairs formed, $\bar{n}$, can be calculated as:

$$
\bar{n}=\frac{E_{d e p}}{W}
$$




\subsection{Gas Multiplication}

Electron or gas multiplication is the phenomenon where primary electrons are accelerated to energies greater than the ionization energy of surrounding neutral gas molecules, resulting in subsequent ionization during a collision. Normally, when a relatively weaker E-field is applied the primary electron and positive ion drift towards the anode and cathode respectively, colliding with atoms in the gas in the process. When the E-field is strong enough so that the electron gains enough kinetic energy between collisions to cause further ionization, additional ion pairs are formed in a process called a Townsend avalanche. As can be deduced, at higher gas pressures the required E-field increases as the distance between collisions is decreased. The equation governing the increase in number of electrons per unit length is given by the Townsend equation [32]:

$$
\frac{d n_{e}}{d x}=n_{e} \alpha
$$

Where $\alpha$ is the Townsend coefficient for a gas (dependent on the E-field strength, gas composition, and pressure), and $\mathrm{n}_{\mathrm{e}}$ is the number of electrons created. For a simple parallel plate geometry where E-field is spatially constant, $\alpha$ is a constant and the solution to the differential equation becomes:

$$
n_{e}(x)=n_{e}(0) e^{\alpha x}
$$


As can be seen, the number of electrons increases exponentially with distance for this case. With more complex E-fields that are not constant such as those in THGEM holes, $\alpha$ frequently changes. To quantify the multiplication of electrons from these Efields, electromagnetic simulation software (such as ANSYS Maxwell) is first used to simulate E-fields. The E-field data can then be used as an input for simulating detector gas multiplication using a particle detector simulator such as Garfield++. Simulation studies have been performed using similar procedures $[33,34]$ and were used for the design optimization of THGEM holes and E-fields throughout this detector.

\subsection{Traditional Proportional Counter and THGEM Comparison}

Traditional proportional counters utilize a wire anode surrounded by a cylindrical or spherical cathode structure (see Figure I.5). As an ionizing particle enters the sensitive volume between the cathode tube or sphere and anode wire, it interacts with the counting gas creating ion pairs. An E-field is applied between the cathode and anode, creating an ion drift region and avalanche region closer to the anode wire. The E-field which must be applied to induce gas multiplication restricts the size of traditional proportional counters, as can be seen in equation 4.1 .

$$
E(r)=\frac{V}{r \ln \left(\frac{a}{b}\right)}
$$

Where $\mathrm{E}(\mathrm{r})$ is the E-field corresponding to the distance $\mathrm{r}$ from the anode, $\mathrm{V}$ is the applied voltage, $\mathrm{a}$ is the anode wire radius, and $\mathrm{b}$ is the cathode tube inner radius. For monitoring large areas, it is desirable to keep $\mathrm{b}$ as large as possible to increase the particle 
interaction volume of the detector, which also requires an increase in voltage to maintain the same E-field strength. Eventually the applied High Voltage (HV) reaches impractical levels, thus limiting the size of the detector.

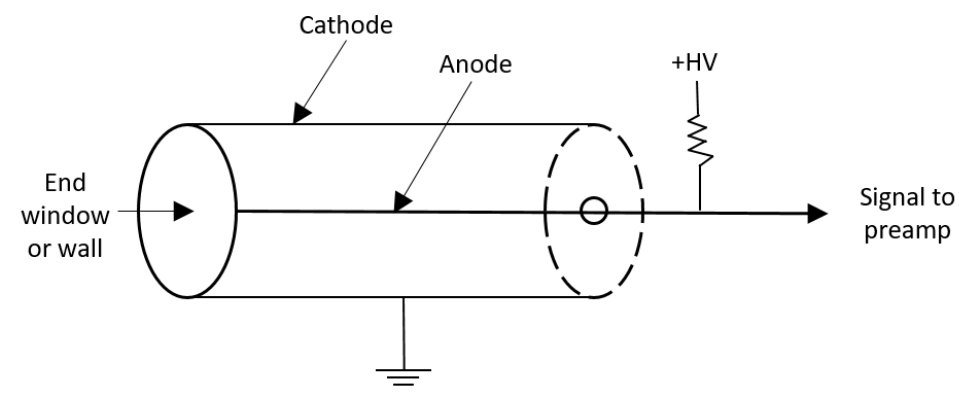

Figure I.5: A simplified illustration of a traditional proportional counter.

In addition to improving the active area of a detector, it is of great importance to keep the electron multiplication as high as possible to extend the low energy cut-off of particles. As previously discussed, low energy beta-ray emitters such as tritium and ${ }^{14} \mathrm{C}$ are by-products in nuclear power generation and are difficult to detect due to their low beta-decay energies. In contrast to traditional proportional counters, THGEMs can offer multiple stages of amplification by cascading them, increasing charge amplification and signal-to-noise ratio in the detector without requiring expensive and specialized electronics. The general layout and functional regions of a THGEM assembly in single and double- THGEM configurations are shown below in Figure I.6. 


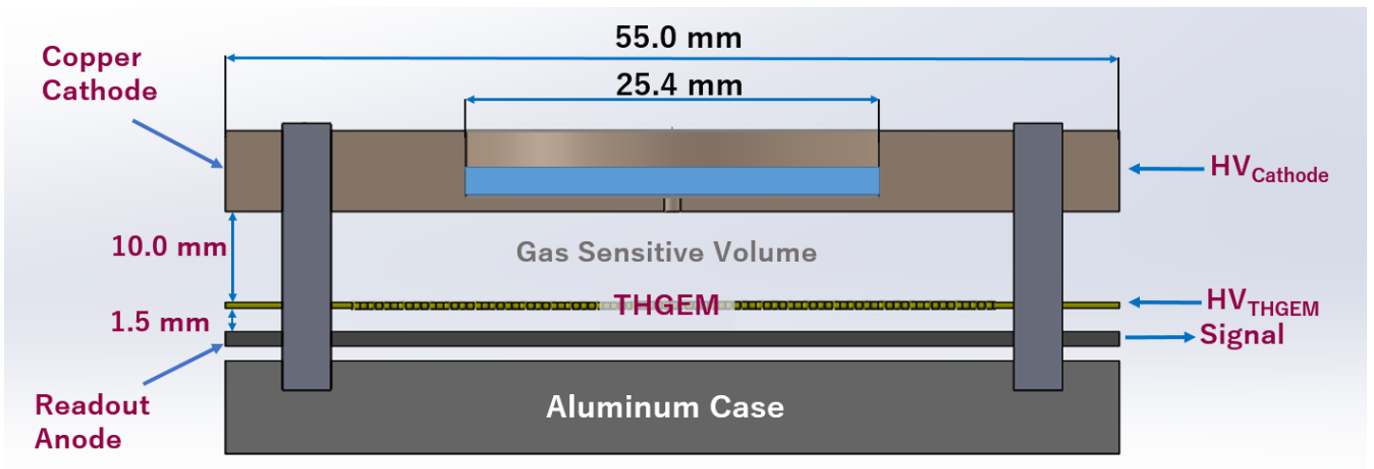

Figure I.6: Overall structure of THGEM assembly with typical spacing dimensions.

The main THGEM components in this thesis were assembled by layering a copper cathode, THGEMs, and a readout board with appropriate spacing between each using insulating spacers on four Teflon screws in a square configuration. A readout board with a hole drilled into each corner is first placed on the screws which are set into tapped holes in an aluminum chamber. The insulated Teflon spacers are then used to support the THGEM slightly above the readout board, followed by a larger gap between the top of the THGEM and copper cathode. Nuts also made of Teflon are then used to secure a lid (not displayed in figure) above the copper cathode.

In order to function as a radiation detector, $\mathrm{HV}$ must be applied across the THGEM, which consists of top and bottom copper clads on each side of an FR-4 insulator as shown in Figure I.7. To accomplish this, voltage dividers were implemented to reduce the number of $\mathrm{HV}$ power supplies required. When an ionizing particle such as a beta-ray enters the sensitive volume situated between the copper cathode and first THGEM, it creates electron-ion pairs in the counting gas. A drift E-field is then used to guide electrons in the direction of the field towards the THGEM holes. Strong E-fields created 
in the holes protrude out into the drift region, focusing the electrons into the multiplication region. Here, the E-field is intense enough to induce electron multiplication. The transfer E-field is used to guide the electron cloud formed after the first stage of multiplication into a second stage of multiplication in a subsequent THGEM. After two stages of multiplication, the final electron cloud is collected on a readout anode at ground voltage.

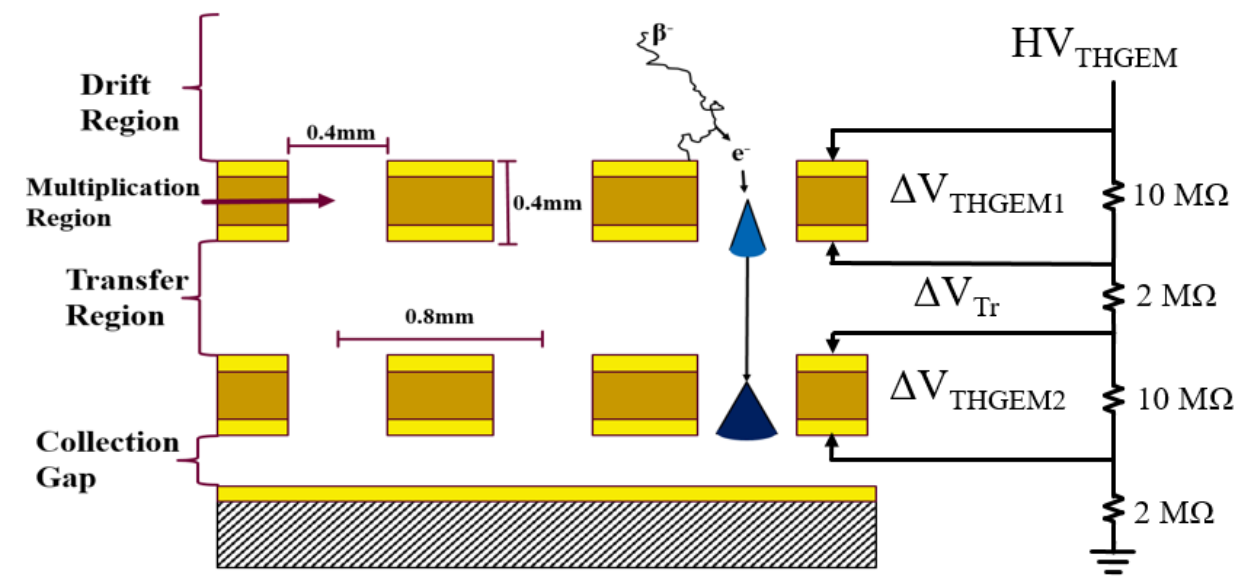

Figure I.7: Functional regions of a THGEM assembly with dimensions of utilized THGEM indicated.

Unlike traditional wire anode proportional counters, THGEM detectors are absolved of the thin wire anode structure which are fragile and much more difficult to assemble. In addition, the spacing between components can be easily modified by adding or removing spacers, and due to the robustness of THGEMs they are not as limited in area as larger traditional proportional counters. Another advantage of THGEMs is their affordable cost ( $\sim 30 /$ unit), and automated fabrication process as a PCB enabling mass manufacturing. Additional stages of multiplication can be added if necessary, although 
the Raether limit governs the maximum achievable gain before discharge occurs due to the high density of electrons in the avalanche volume. For THGEMs the value is typically $10^{7}$ for one electron. A benefit of cascaded THGEMs is their ability to increase the volume of avalanches via diffusion of electrons exiting the holes at each stage (where single THGEMs must be operated at higher $\Delta \mathrm{V}$ to achieve the same gain and results in a greater electron density), thus increasing the limit [35]. Finally, THGEMs used in fixed and portable instruments could be easily replaced compared to wire anode counters, which are often subjected to physical damage requiring repair when used in an industrial setting.

\subsection{Thesis Preface}

The development of a THGEM based beta-ray detector is presented. Chapter 2 will focus more comprehensively on the detector design, and tests using an alpha source for THGEM function tests, E-field optimization, gain stability measurements, effective gain measurements, and to acquire data for subsequent energy calibrations. Chapter 3 of this thesis includes beta-ray spectra measurements and further optimization of the detector for beta-ray sources. This was done in conjunction with Monte Carlo simulations to validate the experimental results. Upon completion of beta-ray spectra analysis, this prototype detector will serve as proof of an advanced THGEM based detector for swipe sample beta-ray contamination measurements. Lastly, chapter 4 will briefly discuss important results, conclude the study, and suggest future research based on this detector. 


\section{Chapter II.THGEM Beta-ray Detector Design and Pulse Processing System}

\subsection{Aluminum Vacuum Chamber}

In order to house the THGEM and other required components in a chamber that can withstand a range of gas pressures, a vacuum chamber was designed based on a previous chamber prototype [12]. Machined from $6061 \mathrm{~T} 6$ aluminum alloy, the walls were designed with an increased thickness to ensure structural integrity when forming a seal. The chamber consists of two parts, a base and a lid, which interface together and formed a hermetic seal using a 7" diameter, 75D Viton O-Ring. The lid of the chamber had holes drilled for two high vacuum 3/8" SHV connectors and one high vacuum 3/8" BNC connector. In addition, a 1/4" hole was drilled in the lid for a gas filling port. Each hole was hermetically sealed by applying Loctite $1 \mathrm{C}$ Hysol epoxy. The technical drawings used to machine the chamber can be seen in Figure II.1 and Figure II. 2 below. 

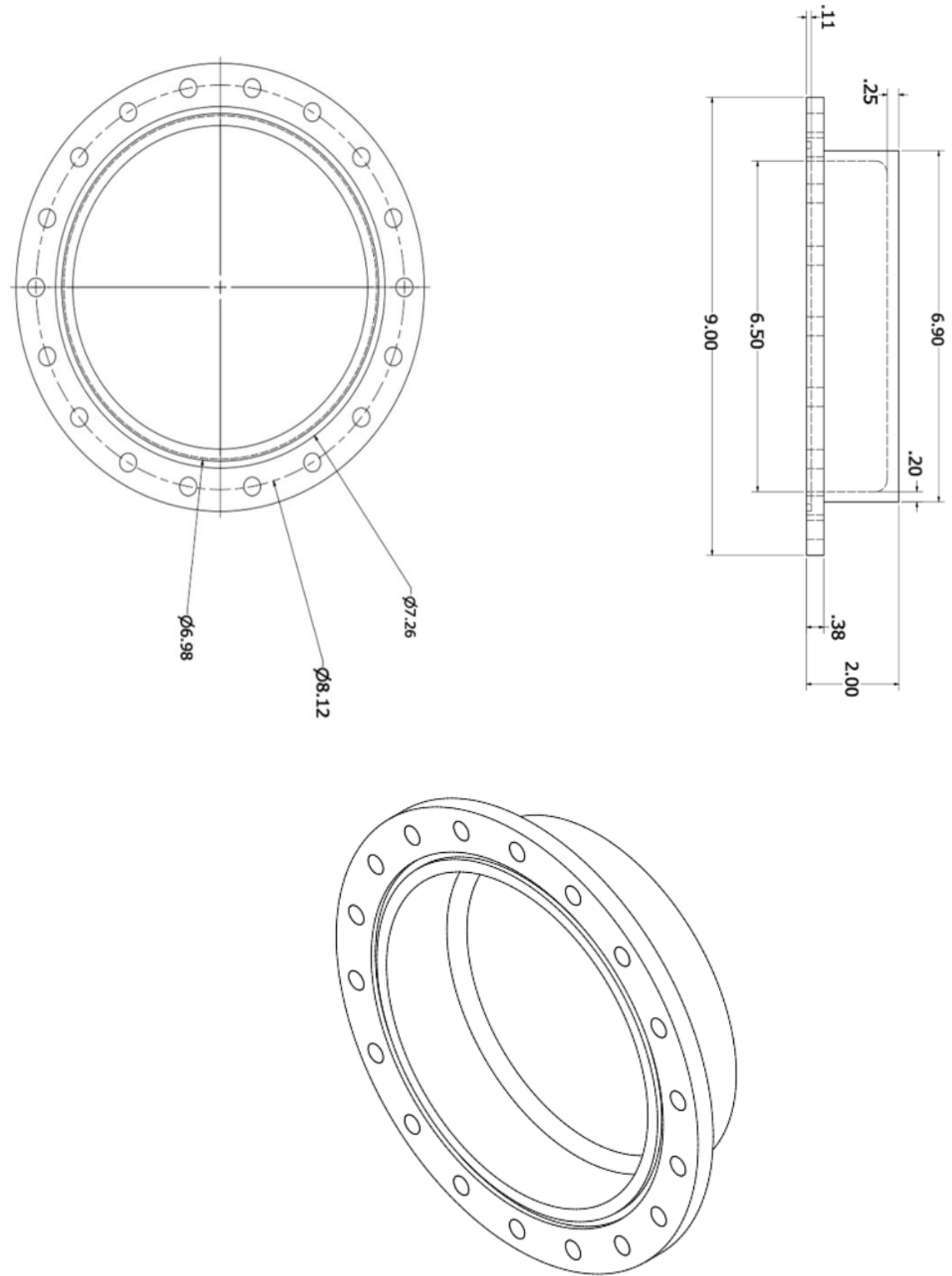

Figure II.1: Technical drawings for base of aluminum vacuum chamber. 

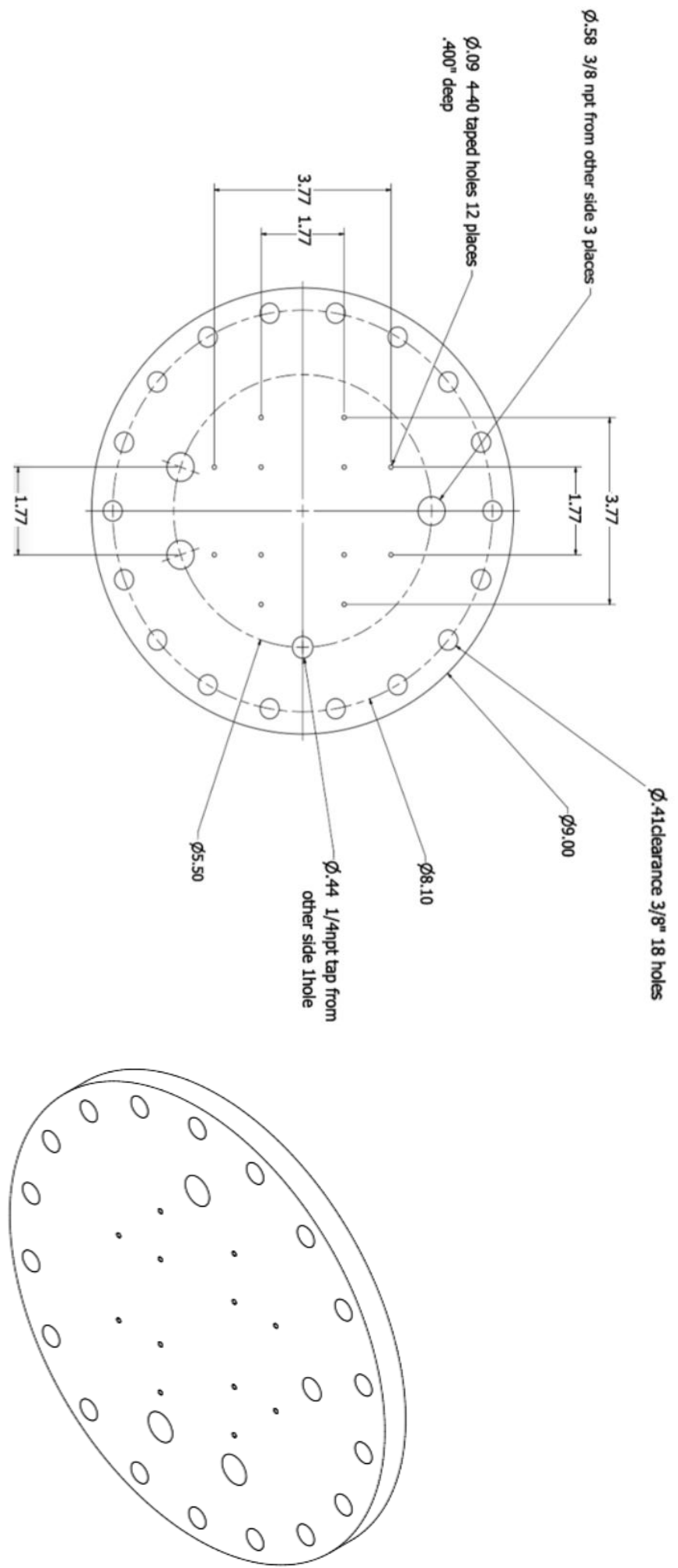

Figure II.2: Technical drawings for lid of aluminum vacuum chamber. 
To prevent Paschen discharges between the SHV high vacuum connectors (often set to greater than $-1000 \mathrm{~V}$ ) and the aluminum case (ground), an insulator was 3D printed using Polylactic Acid (PLA) filament indicated in Figure II.3 below. In addition, heat shrink and kapton tape were used on exposed wires and surfaces such as copper pads on PCBs to mitigate electrical discharge. An extensive effort was made to clean the chamber and electrical components prior to sealing using Kimwipes and isopropyl alcohol to remove contaminants inside the chamber. The entire chamber and detector assembly can be seen in Figure II.4.

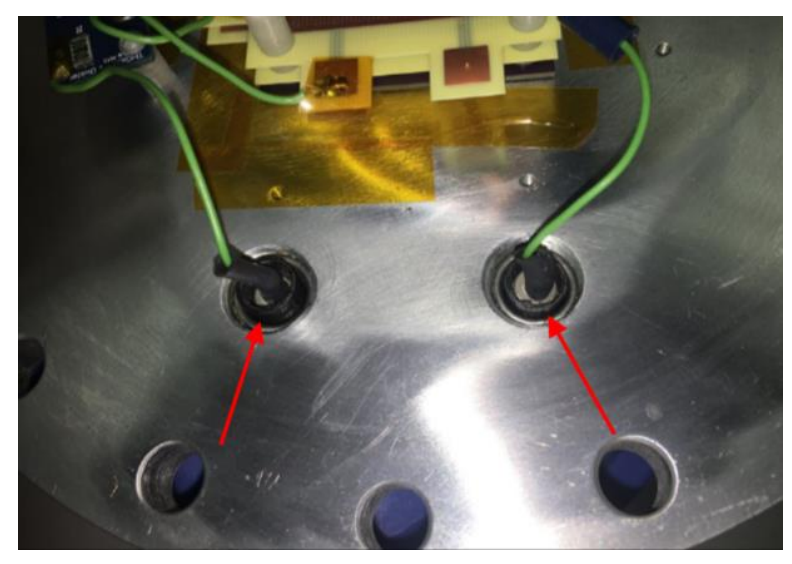

Figure II.3: 3D printed SHV insulators used to prevent discharge between connector and aluminum case. 


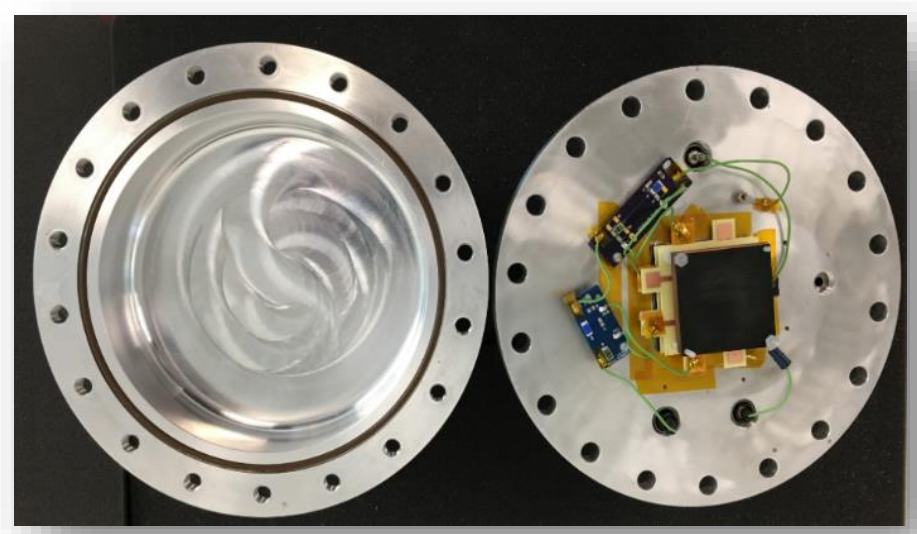

Figure II.4: Aluminum vacuum chamber base and lid with double-THGEM and components installed

Using an oil diffusion pump, the vacuum chamber was evacuated to less than $10^{-4}$ torr and backfilled with counting gas up to pressures of 1 atm (760 torr). After pumping for at least 12 hours to ensure removal of air and other impurities inside the chamber, it was backfilled with either TE-propane or P10 counting gas to the selected pressure following a devised procedure. It should be noted that the first time the chamber is pumped or following any extensive periods of the chamber being exposed to contaminants, the pumping time should be increased to ensure removal of residual impurities. Since one of the main goals of this thesis was to increase the multiplication gain, a counting gas with low electron attachment coefficient and high Townsend coefficient should be used. In addition, a gas with lower $\mathrm{W}$-value is ideal to produce more electron-ion pairs per source particle and increase the SNR. TE-propane has the benefit of more closely modelling muscle tissue for dosimetry and was readily available, thus it was primarily used throughout this thesis. 


\subsection{Copper Collimator for Alpha and Beta Sources}

Two copper source holders were machined to secure the sources above the detector SV and allow particular transmission angles of particles into the SV (see Figure II.5). The copper source holder was machined to hold a disk source with a diameter of $2.54 \mathrm{~cm}$, and collimate source particles to a relatively fixed path length. To accomplish this and also allow a reasonable number of particles through, the collimation hole was drilled with a $2 \mathrm{~mm}$ depth and $1 \mathrm{~mm}$ diameter. For the beta source holder, a larger diameter of 2.26 $\mathrm{cm}$ was drilled to allow for the maximum number of source particles to enter the SV and thus increased efficiency.

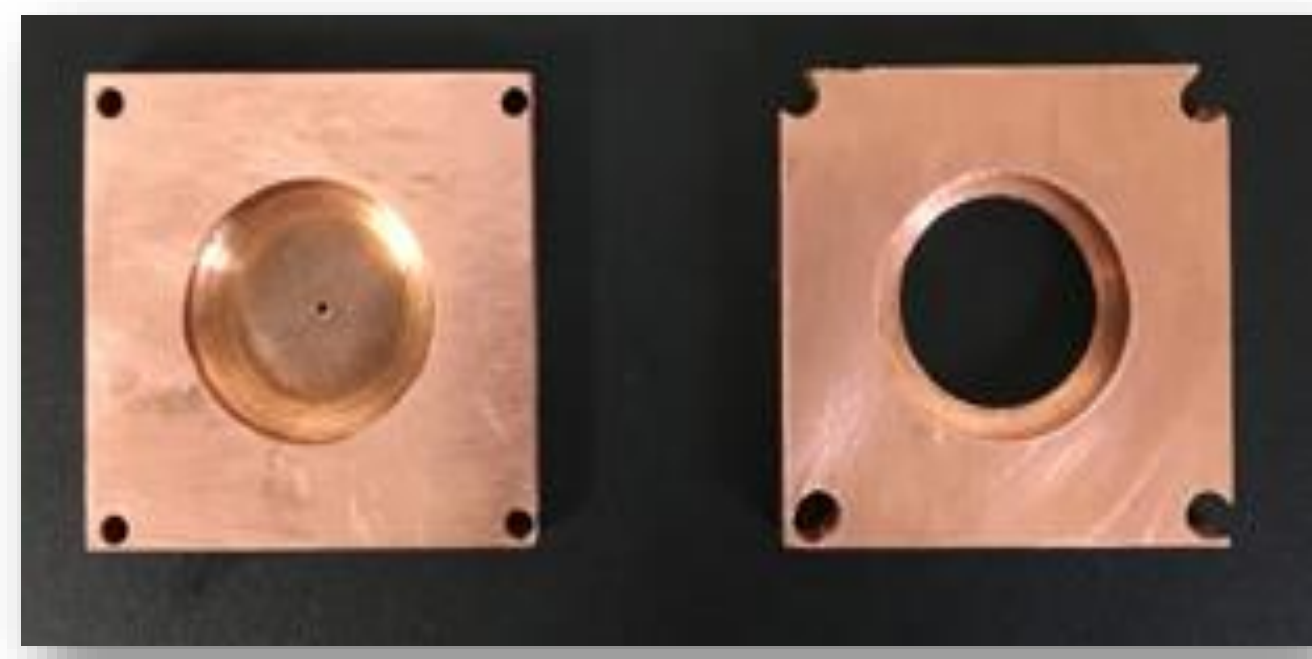

Figure II.5: Alpha (left) and beta (right) copper source holders. 


\subsection{THGEM Designs}

Three THGEM designs were considered for this thesis. Holes were drilled on a $55 \times 55 \times 0.402 \mathrm{~mm}$ board composed of copper layers on FR-4 insulator. Holes were equally spaced across the active surface, each with a diameter of $0.40 \mathrm{~mm}$ and pitch of $0.80 \mathrm{~mm}$. All three THGEMs were tested for functionality, and the first two described below were found to be the most reliable in terms of obtaining a signal and reaching higher operating voltages before discharge occurred. Since the second THGEM listed had a greater SV, it was selected for further study.

Table II.1: THGEMs tested in order of increasing SV for 1cm SV height.

\begin{tabular}{|c|c|c|c||}
\hline & THGEM 1 & THGEM 2 & THGEM 3 \\
\hline $\begin{array}{c}\text { Total SV } \\
\text { Volume }\left(\mathrm{cm}^{3}\right):\end{array}$ & 1.77 & 17.64 & 24.91 \\
\hline
\end{tabular}

All THGEMs were fabricated by Milplex Circuit Inc., which was previously proven to produce the best quality THGEMs compared to other facilities [36]. Due to past efforts failing to produce THGEM holes with concentric rims [12], THGEMs without etched rims were designed (shown in Figure II.6). In addition, it is very important to produce uniform holes with minimal copper debris, which is accomplished by using more precise drilling and polishing, as well as frequent drill bit changes as performed at Milplex. Abrasive pumice particles were used to remove contaminants and leave a uniform copper surface to prevent discharge across the holes that can occur across sharp copper edges, as evident in Figure II.7. 

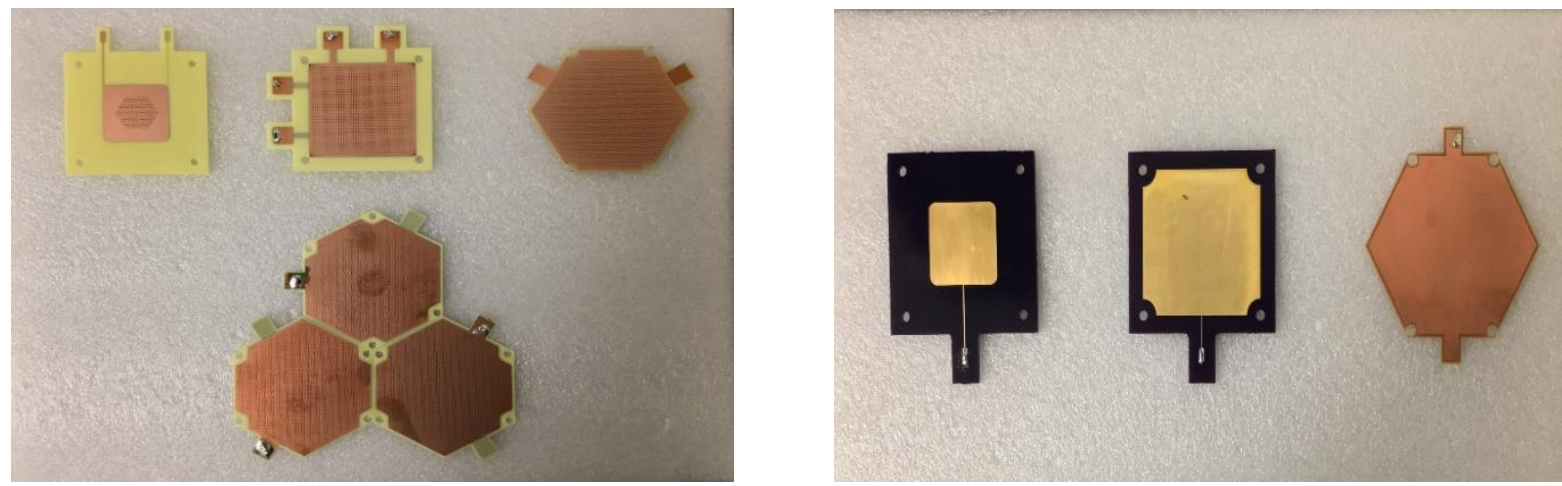

Figure II.6: THGEMs fabricated by Milplex Circuit (Canada) Inc, and Readout Board PCBs fabricated by OSH Park. THGEM 3 can interface well to increase active area as shown due to its hexagonal shape.

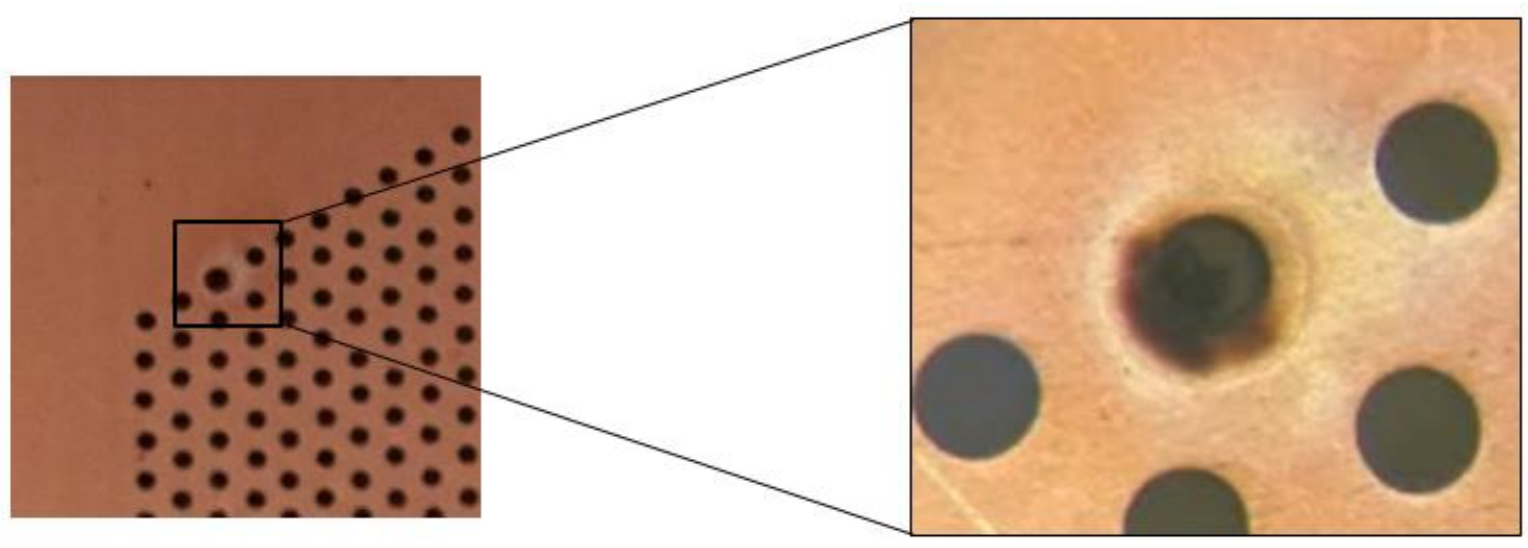

Figure II.7: Image of burnt THGEM hole from electrical breakdown due to a non-uniform copper surface.

\subsection{HV Biasing and Signal Processing}

In order to bias the cathode and THGEMs while minimizing the number of power supplies needed, PCB voltage dividers were employed. Ideally, they should include passive low pass filters to remove noise induced by the HV power supplies using RC filters, however after testing two versions of voltage dividers with passive filters included, 
operation was unreliable as shorting and creepage regularly occurred across the capacitors and resistors due to insufficient clearance. This was confirmed by measuring the resistance across the capacitors using a Multi-Meter, which indicated a measurable resistance when it should not have. In addition, sparking was observed in the newer HV dividers at $\mathrm{HV}_{\text {THGEM }} 800 \mathrm{~V}$ using a transparent chamber base. As a result, older voltage dividers shown on the left in Figure II. 8 without noise filters were used (which had a significantly reduced rate of failure compared to the newer versions). In the future, it is advised to design a HV noise filtering PCB voltage divider with more adequate spacing and circuit components rated for higher voltages to ensure proper function.

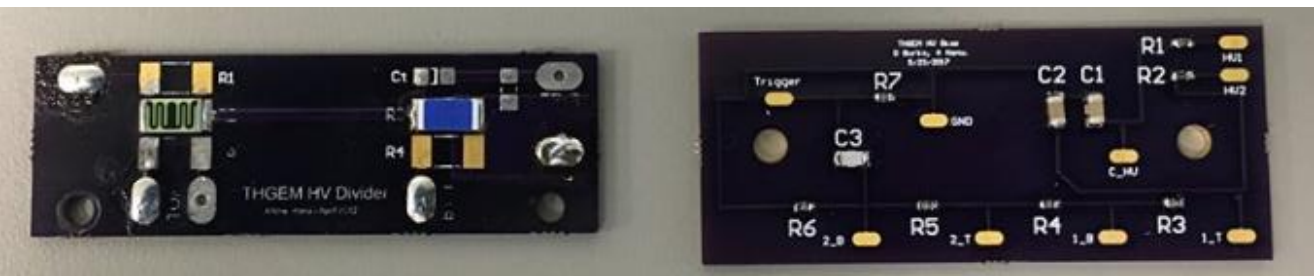

Figure II.8: Example of older voltage divider (left) and newer model (right) with HV noise filtering. The newer model (still under development) will be able to provide noise filtering and bias double-THGEMs and the cathode with one PCB, whereas the older version required two identical PCB dividers in series to bias double-THGEMs.

The dual HV power supply used was a Canberra 3125, containing two units that could separately bias from 0 to $5000 \mathrm{~V}$ of either positive or negative polarity voltage. A negative HV was applied directly to the cathode, and another to the THGEM HV dividers. A schematic of the voltage dividers used is displayed below (see Figure II.9).

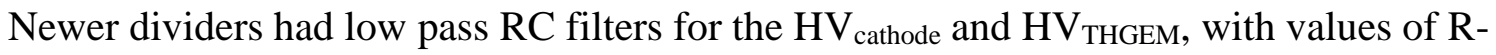
$1=8.2 \mathrm{M} \Omega, \mathrm{C} 1=15 \mathrm{nF}, \mathrm{R} 2=1.0 \mathrm{M} \Omega$ and $\mathrm{C} 2=15 \mathrm{nF}$ as recommended by Hanu [12]. 


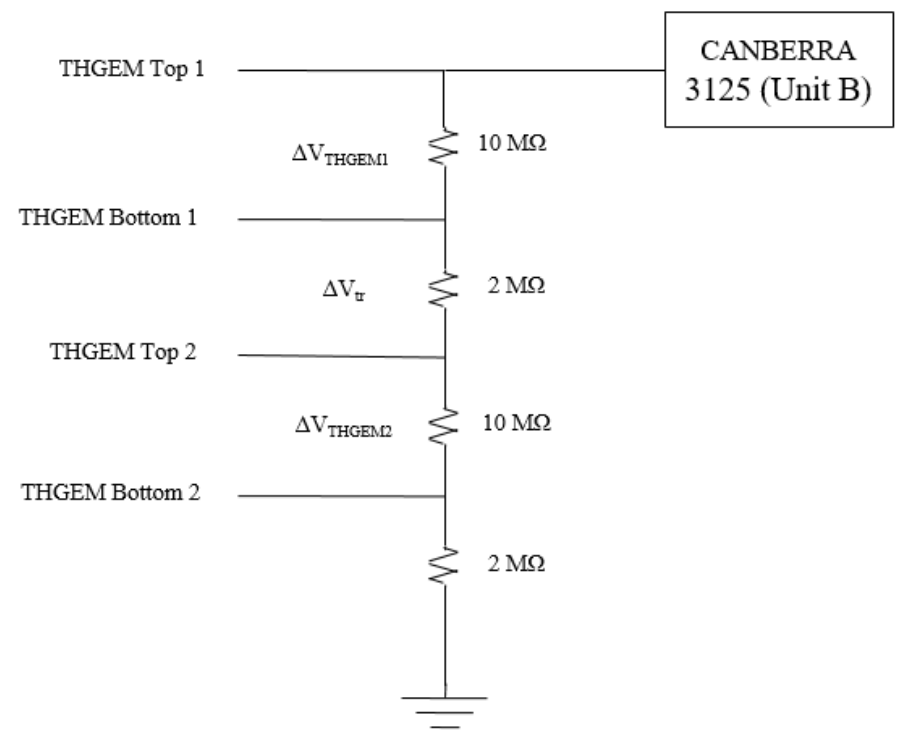

Figure II.9: Voltage divider circuit diagram.

Where:

$$
\Delta V_{\text {THGEM }}=\frac{10 M \Omega}{10 M \Omega+2 M \Omega+10 M \Omega+2 M \Omega}=\frac{5 M \Omega}{12 M \Omega} H V_{T H G E M}
$$

To process each pulse, two preamplifiers and two MCAs were considered. The two preamplifiers tested were an ORTEC 109A and an Amptek A250CF CoolFET. The CoolFET utilizes a thermoelectrically cooled FET which reduces noise levels from the preamplifier. The two ADCs considered were an analogue peak sensing ORTEC 919 Spectrum master (also requiring a shaping amplifier), and DSPEC digital signal processor (which directly digitizes the preamplifier signal).

Using a pulser, the systems were briefly compared in terms of peak resolution, corresponding to lowest electronic noise. As can be seen below in Table II.2, the Analogue ADC (ORTEC Spectrum Master 919) and CoolFET preamp offers superior 
noise reduction. However, due to stability issues with the CoolFET when the detector was connected the Ortec 109a preamp was ultimately used.

Table II.2: Determination of pulse processing electronics with lowest noise levels.

\begin{tabular}{|c|c|c|c|c|}
\hline \multicolumn{5}{|c|}{ Ortec 109a \& Analogue ADC } \\
\hline $\begin{array}{c}\text { Pulser } \\
\text { Amp (V) }\end{array}$ & $\begin{array}{c}\text { Pk Centre } \\
(\mathrm{Ch})\end{array}$ & $\begin{array}{c}\text { FWHM } \\
(\mathrm{Ch})\end{array}$ & $\begin{array}{l}\text { Pk Count Rate } \\
(\mathrm{CPS})\end{array}$ & $\begin{array}{l}\text { relative peak } \\
\text { width }\end{array}$ \\
\hline 0.05 & 172.52 & 2.5 & 100.82 & 0.014 \\
\hline 0.065 & 228.21 & 2.35 & 100.97 & 0.010 \\
\hline 0.075 & 256.31 & 2.49 & 100.95 & 0.010 \\
\hline 0.1 & 358.26 & 2.46 & 101.05 & 0.007 \\
\hline \multicolumn{4}{|c|}{ Average Relative Peak Width: } & 0.010 \\
\hline \multicolumn{5}{|c|}{$\begin{array}{c}\text { CoolFET \& Analogue ADC } \\
\end{array}$} \\
\hline $\begin{array}{c}\text { Pulser } \\
\text { Amp (V) }\end{array}$ & $\begin{array}{l}\text { Pk Centre } \\
\text { (Ch) }\end{array}$ & $\begin{array}{c}\text { FWHM } \\
\text { (Ch) }\end{array}$ & $\begin{array}{c}\text { Pk Count Rate } \\
\text { (CPS) }\end{array}$ & $\begin{array}{l}\text { relative peak } \\
\text { width }\end{array}$ \\
\hline 0.05 & 138.45 & 1.5 & 106.96 & 0.011 \\
\hline 0.065 & 190.49 & 1.37 & 107.67 & 0.007 \\
\hline 0.075 & 221.66 & 1.17 & 107.94 & 0.005 \\
\hline 0.1 & 299.68 & 1.19 & 108.27 & 0.004 \\
\hline \multicolumn{4}{|c|}{ Average Relative Peak Width: } & 0.007 \\
\hline \multicolumn{5}{|c|}{ COolFET \& DSPEC } \\
\hline $\begin{array}{c}\text { Pulser } \\
\text { Amp (V) }\end{array}$ & $\begin{array}{c}\text { Pk Centre } \\
(\mathrm{Ch})\end{array}$ & $\begin{array}{c}\text { FWHM } \\
\text { (Ch) }\end{array}$ & $\begin{array}{c}\text { Pk Count Rate } \\
(\mathrm{CPS})\end{array}$ & $\begin{array}{c}\text { relative peak } \\
\text { width }\end{array}$ \\
\hline 0.04 & 369.76 & 7.38 & 97.7 & 0.020 \\
\hline 0.06 & 554.29 & 9.56 & 97.63 & 0.017 \\
\hline 0.08 & 739.53 & 12.08 & 95.99 & 0.016 \\
\hline 0.1 & 923.83 & 15.19 & 92.02 & 0.016 \\
\hline \multicolumn{4}{|c|}{ Average Relative Peak Width: } & 0.017 \\
\hline
\end{tabular}

The final signal processing system used is shown in a block diagram in Figure II.10. SHV and BNC cables were used to connect equipment contained in NIM bins to the detector assembly as seen in Figure II.11 and Figure II.12. 


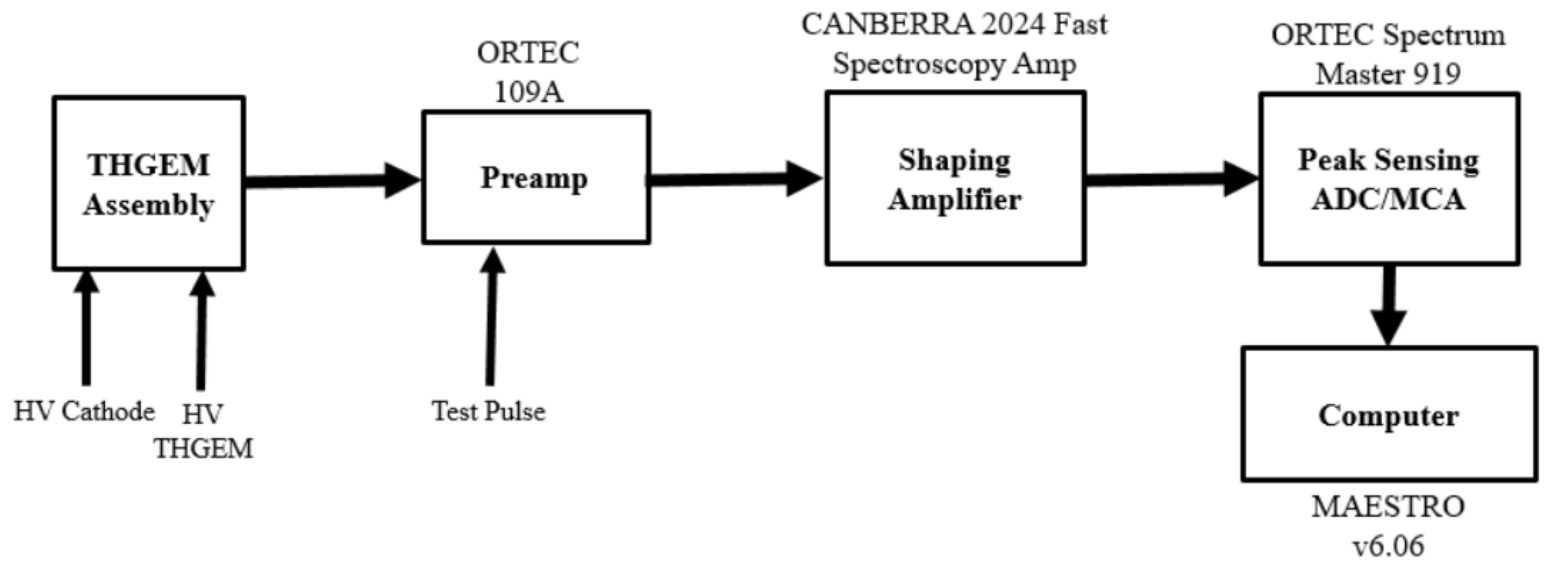

Figure II.10: Block diagram with signal processing equipment used.

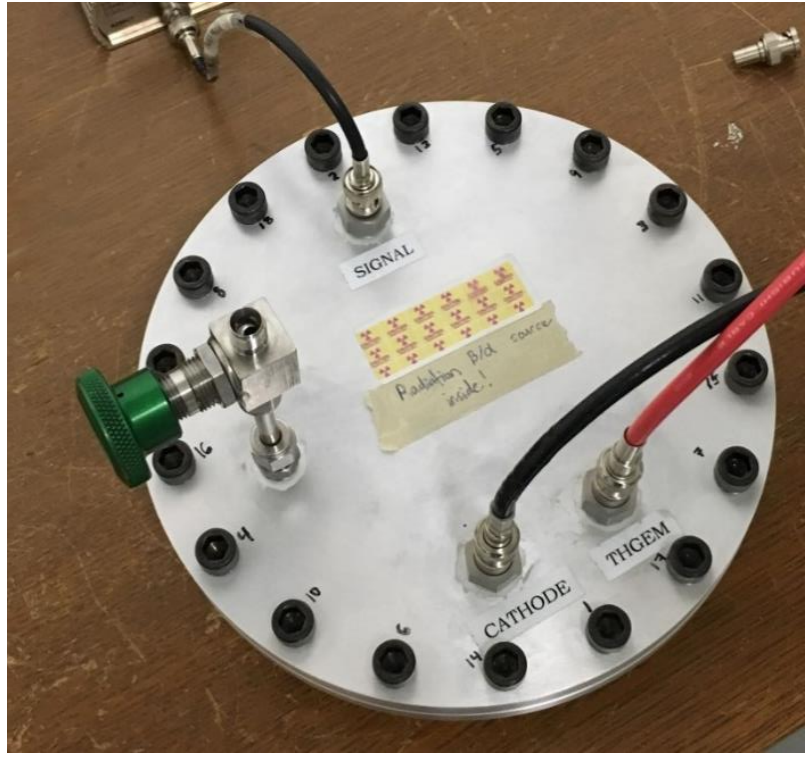

Figure II.11: Lid of aluminum chamber with two high vacuum SHV connectors and one high vacuum BNC connector for signal output to preamplifier.

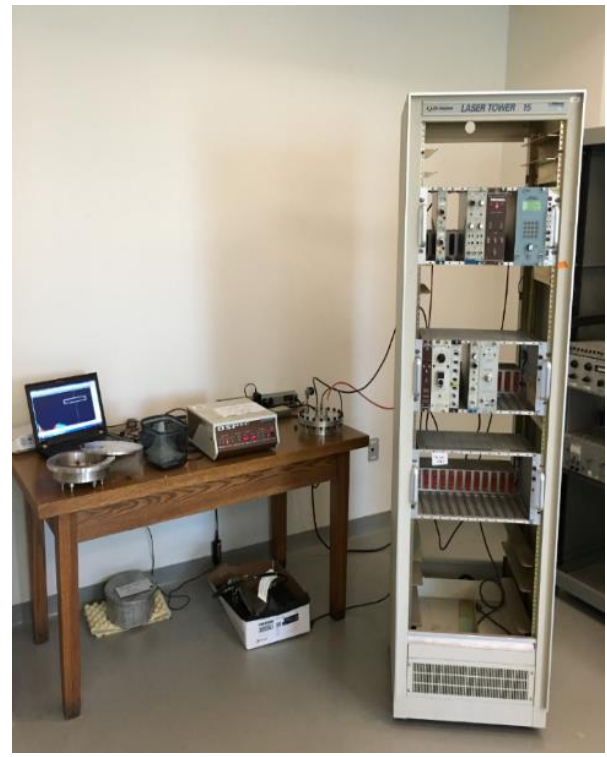

Figure II.12: Experimental layout showing most pulse processing equipment in NIM bins. 


\section{Chapter III.THGEM Beta-ray Detector Performance}

\subsection{Drift E-field Optimization}

As mentioned in Chapter 1 , a ${ }^{244} \mathrm{Cm}$ alpha source was used to optimize the drift Efield, study the long term gain stability, perform effective gain measurements, and eventually energy calibration of beta spectra. During the aforementioned studies, the

${ }^{244} \mathrm{Cm}$ disk source (with active diameter of $5 \mathrm{~mm}$ ) was placed in the alpha source holder containing a $1 \mathrm{~mm}$ hole diameter and $2 \mathrm{~mm}$ depth. Since the alpha particles emitted can be approximated as mono-energetic, and can be collimated to fix the path length of each alpha particle, a roughly constant number of primary electrons can be created using a collimator. By increasing the drift E-field, one can find the ideal HV settings where nearly $100 \%$ of primary electrons are guided into the THGEM holes. The transfer efficiency is given by:

$$
\varepsilon_{t r}=\frac{\text { number of electrons entering hole }}{\text { number of electrons generated in } S V}
$$

With a drift E-field that is too strong, electric field lines do not converge well into the holes resulting in a loss of electrons entering the holes [33]. In addition to this, an Efield that is too weak can result in a loss of electrons to recombination with the fill gas. To find the optimal value for this source, the $\mathrm{HV}_{\text {cathode }}$ was increased and a 10 -minute spectrum for ${ }^{244} \mathrm{Cm}$ was collected. As the drift E-field was increased, more electrons were guided into the holes which was evident as an increase in count rate and peak channel. Eventually, count rate and peak channel would reach maximum values as nearly 
every primary electron was effectively guided into a hole. For 100 torr of TE-propane, the optimal E-drift was found to be $150 \mathrm{~V} / \mathrm{cm}$, as displayed in Figure III.1.

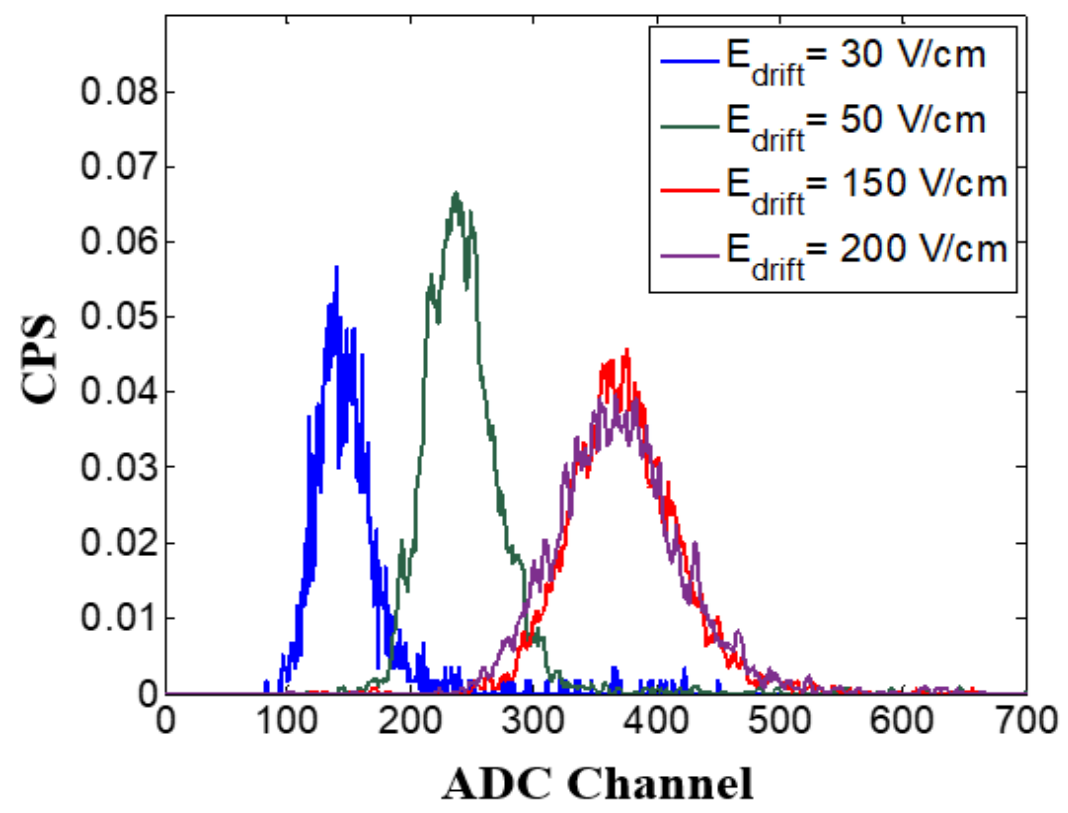

Figure III.1: Drift E-field optimization using ${ }^{244} \mathrm{Cm}$ with a $1 \mathrm{~cm} \mathrm{SV}$ height.

\subsection{Gain Stability}

To measure the effective gain, the ${ }^{244} \mathrm{Cm}$ disk source (with active diameter of $5 \mathrm{~mm}$ ) was used once again and placed in the alpha source holder with $1 \mathrm{~mm}$ hole diameter and $2 \mathrm{~mm}$ depth. A previous THGEM study using a collimator with equal hole diameter and depth reported a high energy tail in the alpha peak due to imperfect collimation [12]. Although the goal was to obtain a more standard Gaussian shape in this thesis, a slight high energy tail was still observed. Thus, in order to accurately measure the peak channel of each alpha spectrum, an exponentially modified Gaussian was fit to each peak. 


$$
y(x)=y_{0}+\frac{A}{2 t_{0}} \exp \left(\frac{\sigma^{2}}{2 t_{0}^{2}}+\frac{x_{c}-x}{t_{0}}\right)\left(1+\operatorname{erf}\left(\frac{x-x_{c}}{\sigma \sqrt{2}}-\frac{\sigma}{t_{0} \sqrt{2}}\right)\right)
$$

This was necessary because despite efforts to collimate alpha particles to a fixed path length, certain alpha particles from the isotropic disk source travelled longer distances in the SV resulting in a greater deposition of energy manifesting as a tail in the alpha peak. Alpha spectra were collected for several hours over 20-minute intervals. An example of one alpha spectrum acquired using MAESTRO and a corresponding MCNP6 simulations for the machined collimator is shown below.
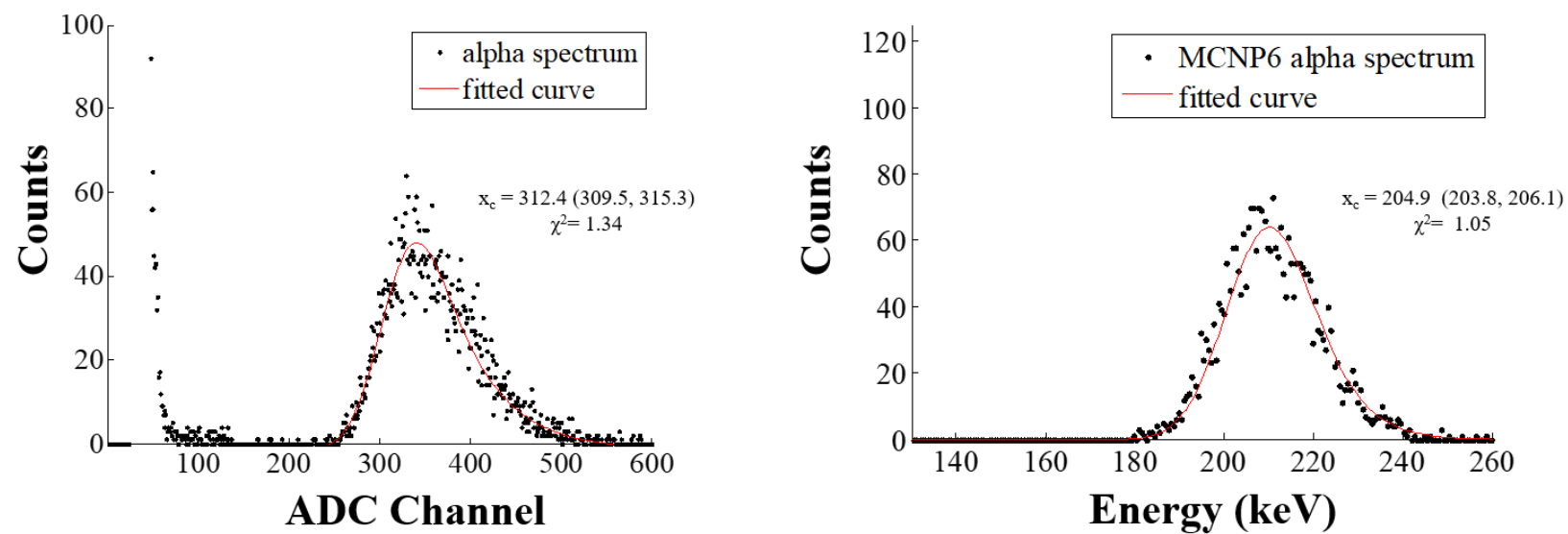

Figure III.2: Experimental and simulated ${ }^{244} \mathrm{Cm}$ spectrum, both fit with exponentially modified Gaussian function with $95 \%$ confidence bounds. Experimental spectra measured at 100 torr TE-propane gas using single-THGEM. 


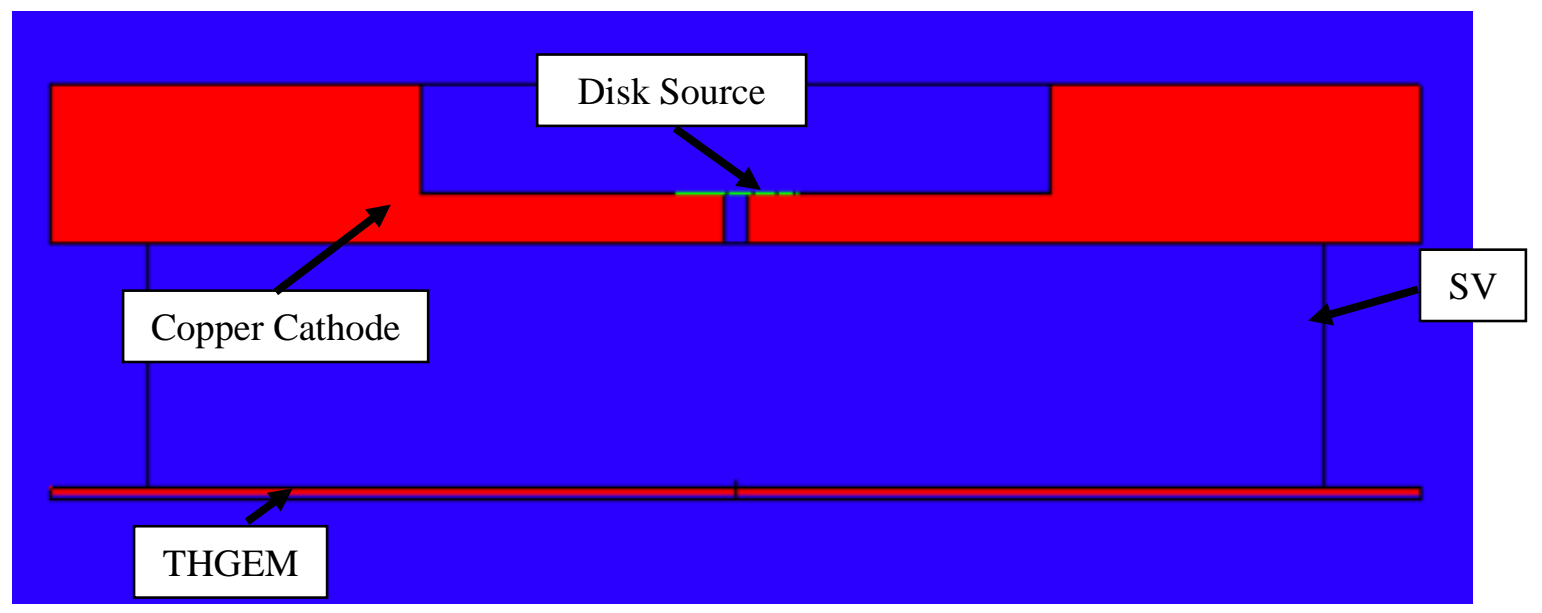

Figure III.3: MCNP6 detector with source geometry in green for $2 \times 1 \mathrm{~mm}$ collimator. $5 \mathrm{~mm}$ diameter disk source with isotropic emission was modelled.

After fitting the collected alpha spectra, the detector gain stability was observed by plotting the peak centre versus time. Any decrease or increase in gain over time would result in a peak shift to the left or right respectively. Overall, there were no drastic changes in peak channel observed over time or any noticeable trend showing an increase or decrease in gain. 


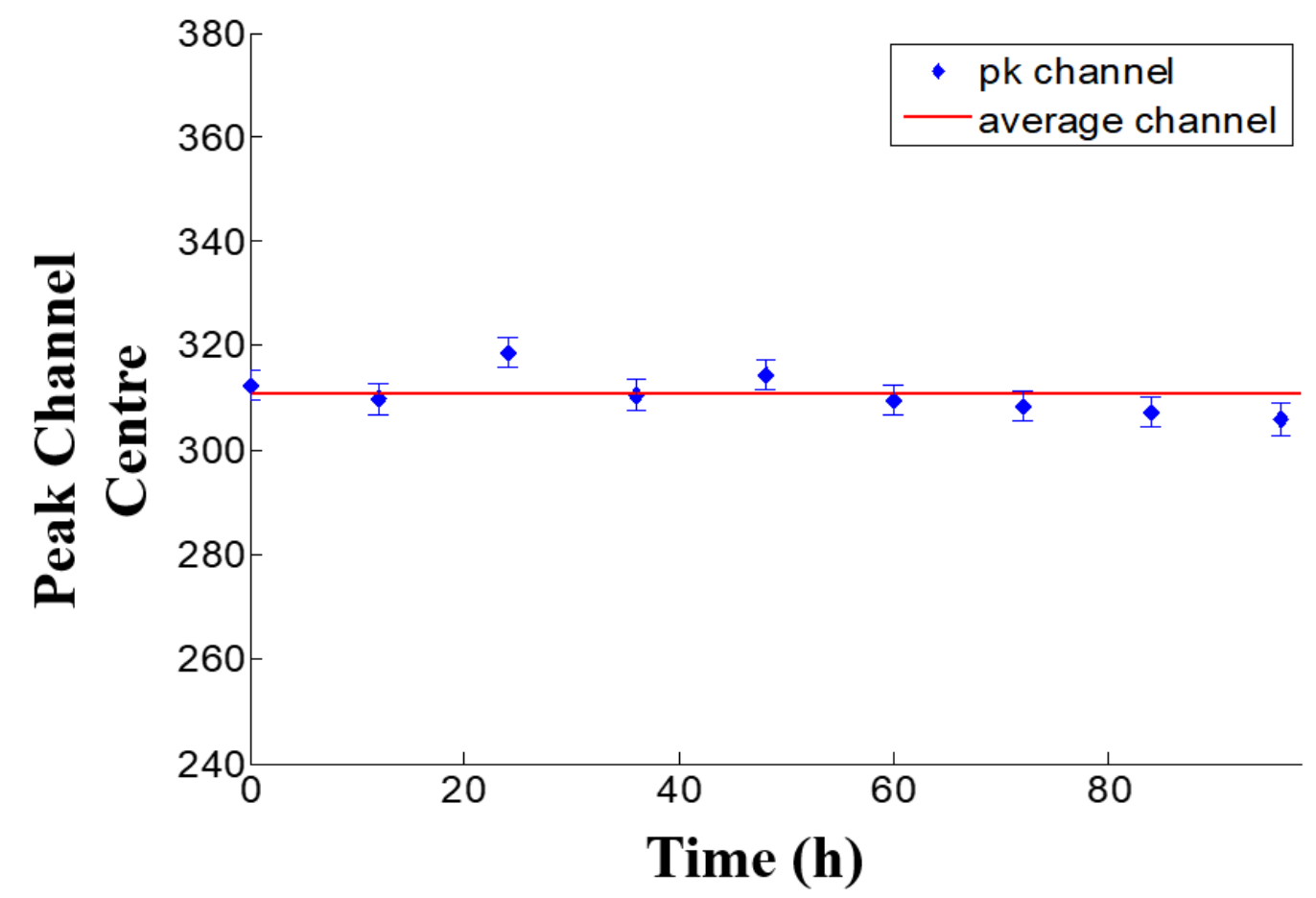

Figure III.4: Long term ${ }^{244} \mathrm{Cm}$ stability for single-THGEM in 100 torr TE-propane gas.

\subsection{Effective Gain}

To benchmark the effective gain of the $42 \times 42 \mathrm{~mm}^{2}$ THGEM for different spacings and fill gases, the effective gain was measured using the ${ }^{244} \mathrm{Cm}$ alpha source. The effective gain is defined as:

$$
\text { Effective Gain }=\frac{\text { Charge Collected }}{\text { Initial Charge Created }}
$$

For single- and double-THGEM configurations, $\Delta \mathrm{V}_{\text {THGEM }}$ was increased and the amplifier gain was set such that the alpha peak was above high frequency noise levels and easily discernable on MAESTRO. As the multiplication gain increased, it was necessary 
to decrease the amplifier gain as the alpha peak went off scale for a 1024 channel conversion gain. A typical HV run for a fixed amplifier gain can be seen below.

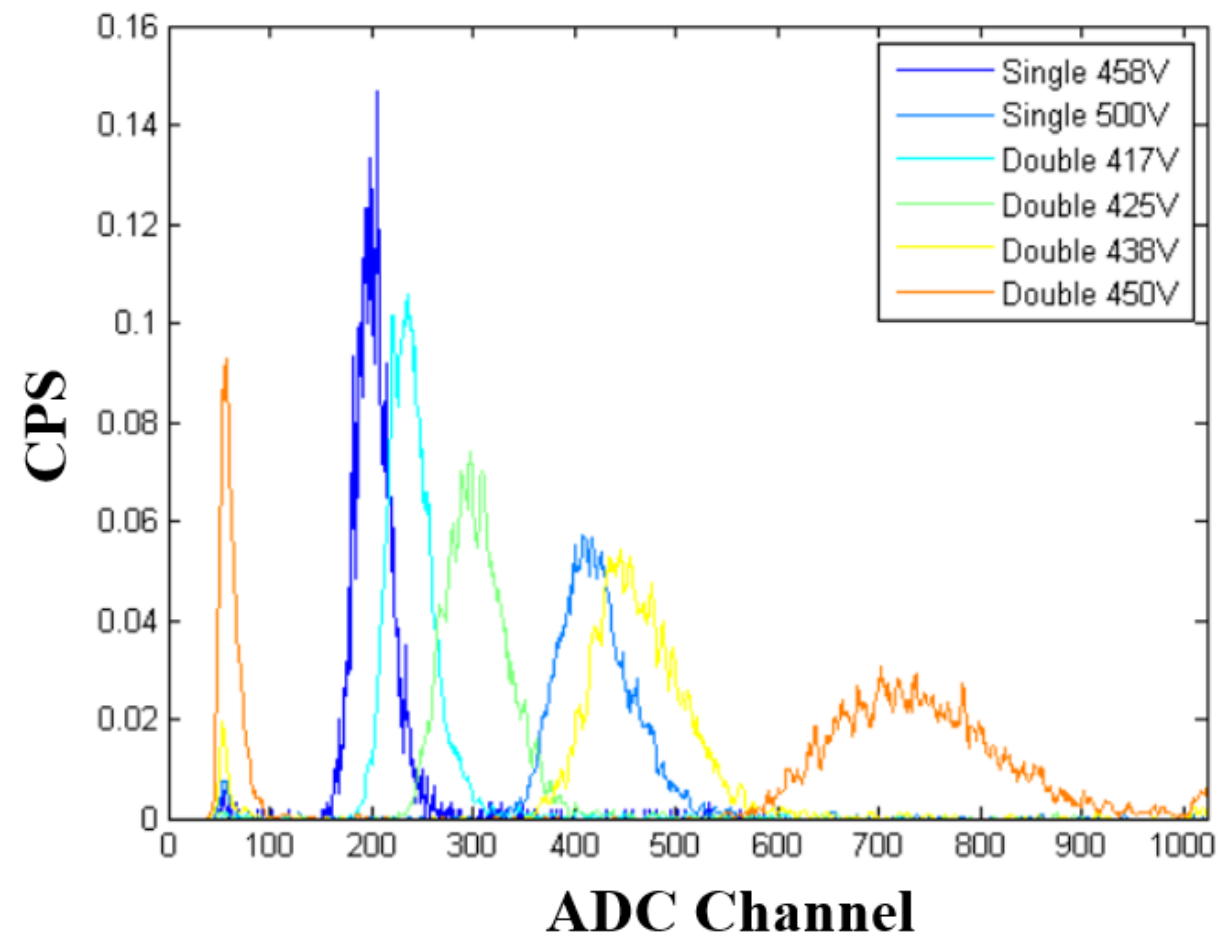

Figure III.5: A set of ${ }^{244} \mathrm{Cm}$ spectra for single- and double-THGEM configurations at 100 torr TE-propane.

The initial charge or number of electrons created was found using the average number of ion pairs created $(\bar{n})$, calculated from $\mathrm{E}_{\mathrm{dep}}$ and each gas's W-value (see Table III.1). After fitting each spectrum, a pulser was connected to the preamplifier test input circuit to find the pulser amplitude voltage corresponding to the peak channel for the set amplifier gain using a pulser calibration equation (see Figure III.6). Once the corresponding pulser amplitude voltage was known, the amount of charge collected on 
the ORTEC 109a preamplifier test capacitor $\left(\mathrm{C}_{\mathrm{t}}=1 \mathrm{pF}\right)$ could be found using the following equation:

$$
\text { number of electrons collected }=\frac{C_{t} V_{t}}{e}
$$

It can also be seen below by the negligible change in slope and channel offset that frequent calibration is not necessary for future measurements if the ADC zero setting is not changed on the ORTEC 919 Spectrum Master.
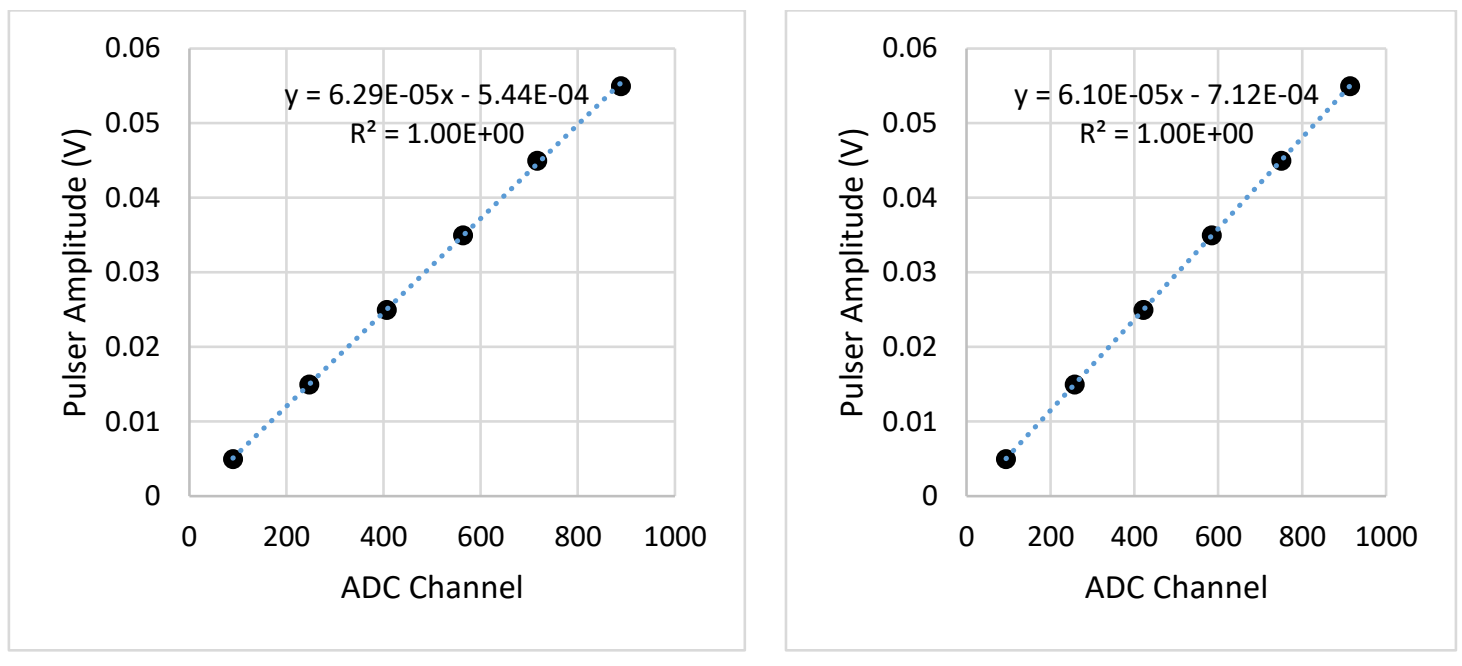

Figure III.6: Pulser calibration with two sets of measurements taken approximately 1 month apart to check for reproducibility, for amplifier gain of 90× using ORTEC 109a test input.

In order to find the initial charge created by the alpha particle, the $\mathrm{W}$-value of the gas and $\mathrm{E}_{\mathrm{dep}}$ are needed. Published studies have found the $\mathrm{W}$-values for TE-propane and P10 (for alpha particles of approximately $5 \mathrm{MeV}$ ) to be $28.0 \mathrm{eV}$ and $26.3 \mathrm{eV}[37,38]$. $\mathrm{E}_{\mathrm{dep}}$ was calculated using NIST astar stopping power for alpha particles with $5.794 \mathrm{MeV}$ in addition to MCNP simulations, assuming particles travel the length of the SV in a straight 
line. It should be noted that at a $1 \mathrm{~cm} \mathrm{SV}$ length for P10, the preamplifier saturated due to high electron multiplication for double-THGEM, and thus despite using the lowest gain settings ( $1 \mathrm{x}$ on preamp, $3 \mathrm{x}$ on amplifier) the peak was not obtainable. This was confirmed by viewing the oscilloscope preamplifier output. Thus, to decrease the magnitude of the signal the SV length was reduced to $0.5 \mathrm{~cm}$. The reduction in length resulted in a decrease in created charge which was accounted for when calculating $E_{\text {dep. }}$ A summary of the results for $\mathrm{E}_{\mathrm{dep}}$ for both fill gases can be seen below, which were calculated using MCNP 6 f8 energy deposition tally in addition to the NIST astar stopping power database [39]:

Table III.1: Properties for two fill gases used for $\mathrm{E}_{\alpha}=5.794 \mathrm{MeV}$.

\begin{tabular}{|c|c|c|c|}
\hline & & $\begin{array}{c}\text { TE-propane, } 1 \mathrm{~cm} \mathrm{SV} \\
\text { length }\end{array}$ & P10, $0.5 \mathrm{~cm}$ SV length \\
\hline \multicolumn{2}{|c|}{ Composition } & $\begin{array}{c}55 \% \mathrm{C}_{3} \mathrm{H}_{8}, 40 \% \mathrm{CO}_{2}, 5 \% \\
\mathrm{~N}_{2}\end{array}$ & $90 \% \mathrm{Ar}, 10 \% \mathrm{CH}_{4}$ \\
\hline \multicolumn{2}{|c|}{ Density at NTP $\left[\mathrm{g} / \mathrm{cm}^{3}\right]$} & 0.001826 & 0.001562 \\
\hline \multicolumn{2}{|c|}{ W-value [eV] } & 28.0 & 26.3 \\
\hline \multicolumn{2}{|c|}{$\begin{array}{c}\mathrm{S}(\mathrm{E}=5.794 \mathrm{MeV})[\mathrm{MeV} \\
\left.\mathrm{cm}^{2} / \mathrm{g}\right]\end{array}$} & 839.70 & Not avail. \\
\hline \multirow{2}{*}{$\mathbf{E}_{\text {dep }}[\mathrm{keV}]$} & MCNP 6 & $204.2 \pm 0.1$ & $109.2 \pm 0.1$ \\
\hline & NIST astar & 201.75 & Not avail. \\
\hline \multicolumn{2}{|c|}{ \# of ion pairs created } & $7205 \pm 4$ & $4152 \pm 4$ \\
\hline
\end{tabular}

Utilizing these values for 100 torr of TE-propane and P10, the initial number of charges were calculated and the effective gain was plotted on a log plot as depicted below: 


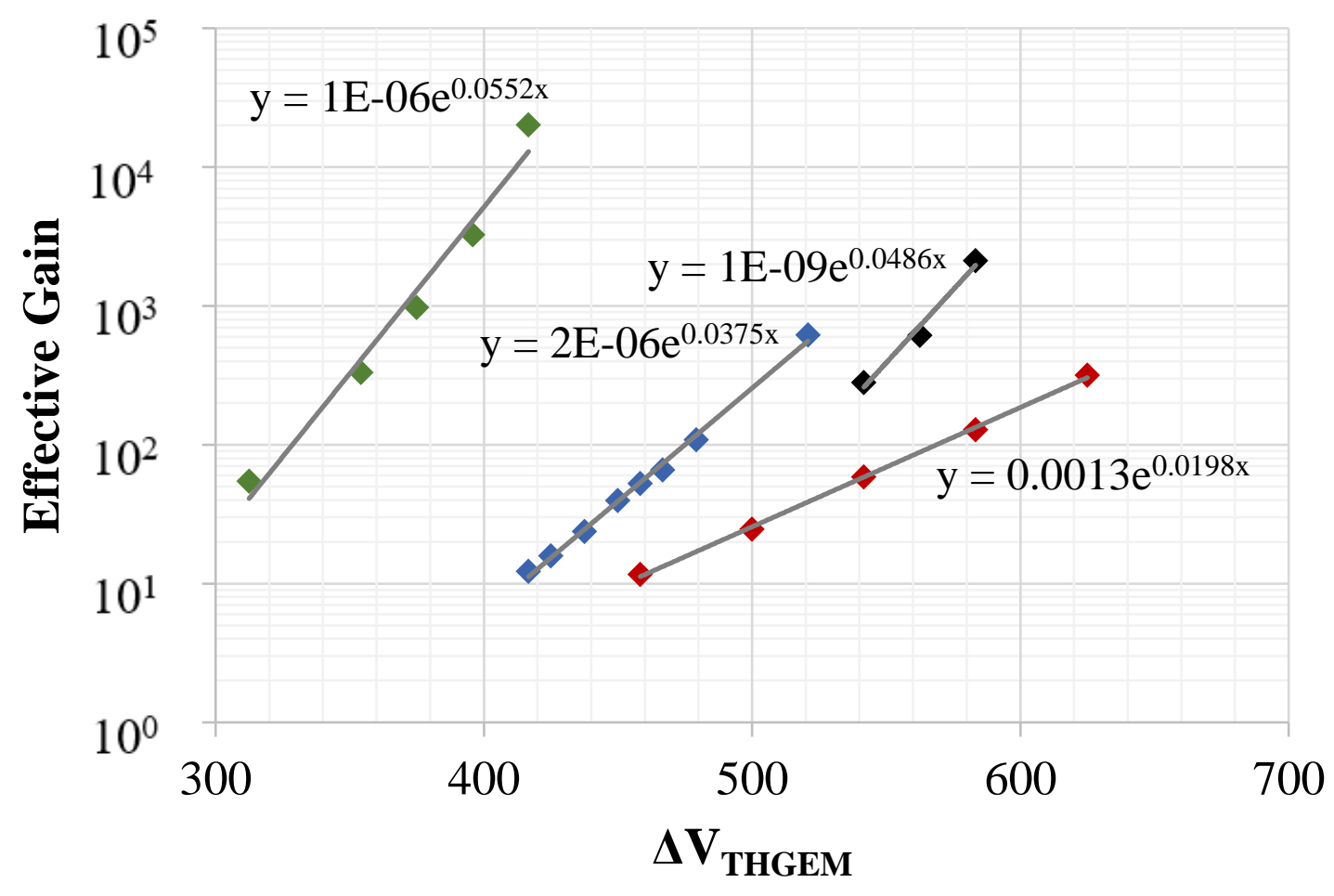

Double-THGEM TE-propane

- Single-THGEM TE-propane

- Double-THGEM TE-propane (increased spacing)

Double-THGEM P10 (increased spacing)

Figure III.7: Effective gain curves for 100 torr TE-propane and P10 using ${ }^{244} \mathrm{Cm}$. Transfer region and collection gap were increased from 3 to $4.5 \mathrm{~mm}$ and 1.5 to $3 \mathrm{~mm}$ respectively to increase maximum operating voltage before sparking was induced.

As can be seen, P10 gas is able to achieve a significantly higher signal than TEpropane gas before electric discharge occurs. It is believed that this can be attributed to two factors; the electron attachment coefficient of the gases affecting the electrons as they drift through the SV, and the Townsend coefficient described in Chapter 1. As mentioned by Dinner [40], electron attachment (and thus reduction in signal strength) is significantly enhanced when $\mathrm{CO}_{2}$ is mixed with $\mathrm{N}_{2}$ with very small $\mathrm{O}_{2}$ impurities as in the case with 
TE-propane. It is noted without $\mathrm{CO}_{2}$, nitrogen itself does not result in significant electron attachment. Only $0.1 \%$ contamination of $\mathrm{O}_{2}$ in $\mathrm{CO}_{2}$ has shown approximately $10 \%$ loss of drifting electrons per $\mathrm{cm}[41]$. Orchard and Waker [42], also noted that the increase in transit time of $\mathrm{Ar}+\mathrm{CO}_{2}\left(90 \% \mathrm{Ar}, 10 \% \mathrm{CO}_{2}\right)$ compared to $\mathrm{P} 10$ gas, resulting in a decrease in signal amplitude and loss of count rate, was attributed to $\mathrm{CO}_{2}$.

Townsend coefficient values were not available for TE-propane and P10 under exact operating conditions. Parameters used to calculate $\alpha / \mathrm{p}$ were experimentally found for uniform high E-fields under low pressures by Sharma and Sauli [43], and although THGEM holes contain non-uniform fields it is still a useful comparison between the gases. Measurements were done using a parallel plate geometry, with a $9 \mathrm{~cm}$ drift region and $0.25 \mathrm{~cm}$ amplification gap. It should be noted the maximum reduced E-field (E/p) reached was approximately $30 \mathrm{~V} \mathrm{~cm}^{-1}$ torr $^{-1}$, resulting in an $\alpha / \mathrm{p}$ value of $0.0148 \mathrm{~cm}^{-1}$ torr $^{-1}$ where a separate study used to compare has reached around $100 \mathrm{~V} \mathrm{~cm}^{-1}$ torr $^{-1}$ with $\alpha / \mathrm{p}$ value of about $2 \mathrm{~cm}^{-1}$ torr $^{-1}$. Both results followed the same trend, however the comparison study was extended to even higher E-fields such as those encountered in THGEMs. However, this second data set was only available for the $\mathrm{Ar}+\mathrm{CO}_{2}$ mixtures. Thus, for $\alpha$ comparison purposes for the two gases used in this thesis, the results for two data sets by Sharma and Sauli using $\mathrm{CO}_{2}$ and $\mathrm{CH}_{4}$ in $\mathrm{Ar}$ are included below:

Table III.2: Comparison of first Townsend coefficient for two gases at low pressure and high E-field, where $\frac{\alpha}{p}=A e^{-B p / E}$

\begin{tabular}{|c|c|c|c|c|c|}
\hline Fill Gas & $\begin{array}{c}\text { \% of gas } \\
\text { in Ar }\end{array}$ & $\begin{array}{c}\mathrm{A}\left[\mathrm{cm}^{-1} \text { torr }^{-}\right. \\
\left.{ }^{1}\right]\end{array}$ & $\begin{array}{c}\mathrm{B}\left[\mathrm{V} \mathrm{cm}^{-1} \text { torr }\right. \\
1]\end{array}$ & $\mathrm{E} / \mathrm{p}\left[\mathrm{V} \mathrm{cm}^{-1}\right.$ torr $\left.^{-1}\right]$ & $\begin{array}{c}\alpha / \mathrm{p}[\text { ion pair cm } \\
\text { torr }\end{array}$ \\
\hline \hline $\mathrm{Ar}+\mathrm{CO}_{2}$ & 22.8 & 221.2 & 207.6 & 21.6 & 0.014816056 \\
\hline $\mathrm{Ar}+\mathrm{CH}_{4}$ & 16.8 & 2.7 & 81.7 & 19.7 & 0.042682924 \\
\hline
\end{tabular}


As previously discussed, TE-propane contains $55 \% \mathrm{C}_{3} \mathrm{H}_{8}, 40 \% \mathrm{CO}_{2}$ and $5 \% \mathrm{~N}_{2}$. and $\mathrm{P} 10$ gas contains $90 \%$ Ar with $10 \% \mathrm{CH}_{4}$. It can be noted from Table III.1 that $\mathrm{CO}_{2}$ has lower electron multiplication than $\mathrm{CH}_{4}$ for almost exact E-field values, which contributes to the higher gain in P10 gas.

\section{4 ${ }^{14} \mathrm{C}$ Disk Source Beta-ray Spectra and Counting Curves}

After optimizing the detector's parameters using an alpha source, a ${ }^{14} \mathrm{C}$ disk source was installed with the beta source holder/cathode. The purpose of the source was to test the detector's response to beta particles, which are much less densely ionizing than alpha particles and require greater electron multiplication to detect. The disk source models as a swipe sample, which can collect removable contamination from surfaces during routine contamination monitoring. Since removable contamination can be internally deposited resulting in greater doses than external contamination, it is of much greater concern and has lower limits in $\mathrm{Bq} / \mathrm{cm}^{2}$ than fixed contamination levels. Following measurements of

${ }^{14} \mathrm{C}$, two other beta-emitting disk sources, ${ }^{90} \mathrm{Sr} / \mathrm{Y}$ and ${ }^{3} \mathrm{H}$, with relatively higher activities were measured. Since the window composition and/or source nominal activities were not accurately known for each disk source, the expected count rate was measured using a traditional Windowless Proportional Counter (WPC) run by the McMaster Health Physics Department to benchmark the THGEM based beta-ray detector's count rates. The signal output waveforms and MAESTRO spectra are included in Figure III.8 and Figure III.9. 


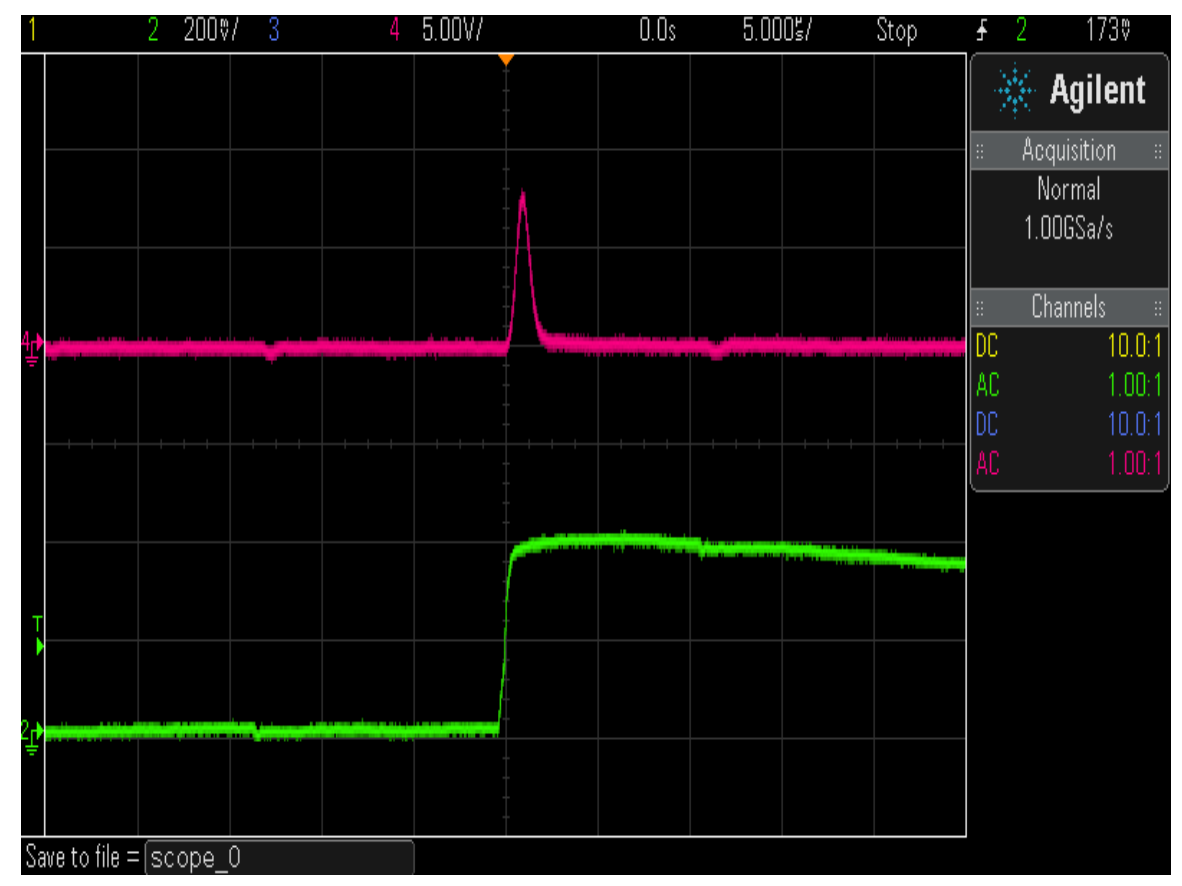

Figure III.8: Preamplifier (200 mV/div) and shaping amplifier (5.00 V/div) oscilloscope output for ${ }^{14} \mathrm{C}$.

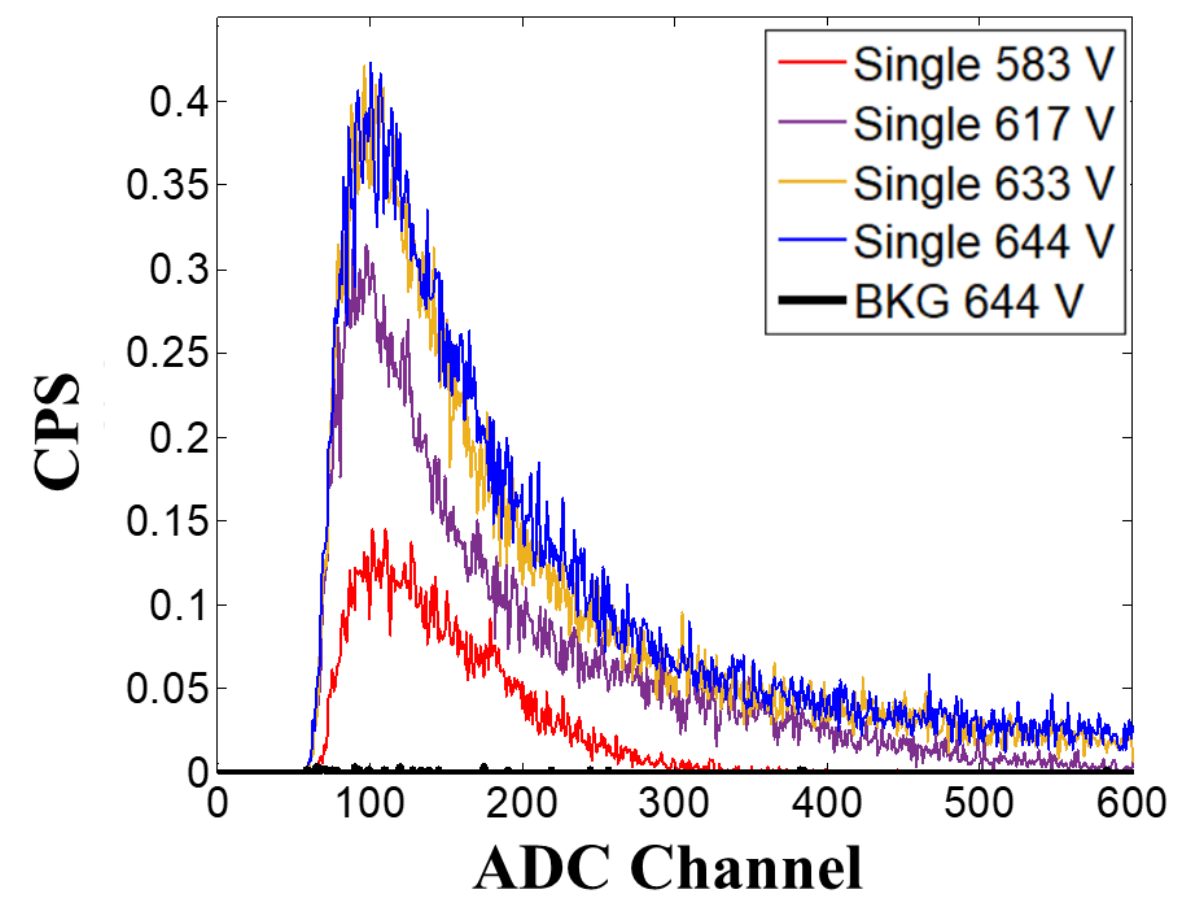

Figure III.9: Set of ${ }^{14} \mathrm{C}$ spectra for HV run for single-THGEM 100 torr TE-propane 
Included below are the counting curves for single and double-THGEM configurations in TE-propane gas, in addition to a double THGEM P10 measurement. These display the net count rate's relationship with applied HV. All measurements were conducted using a $42 \times 42 \times 10 \mathrm{~mm}^{3} \mathrm{SV}, 4.5 \mathrm{~mm}$ transfer region, and $3 \mathrm{~mm}$ collection gap. Using an oscilloscope and test pulser, the LLD was set slightly above background noise levels, which was shown to increase with applied HV. Measurement live time was set to 10 minutes, and spectra were collected at increasing $\Delta \mathrm{V}_{\text {THGEM }}$ until discharge occurred. As can be seen, the P10 gas displayed superior performance to TE-propane gas, in agreement with ${ }^{244} \mathrm{Cm}$ effective gain measurements. Ideally, the detector should operate at the midpoint of the counting plateau to ensure greater stability in count rate measurements.

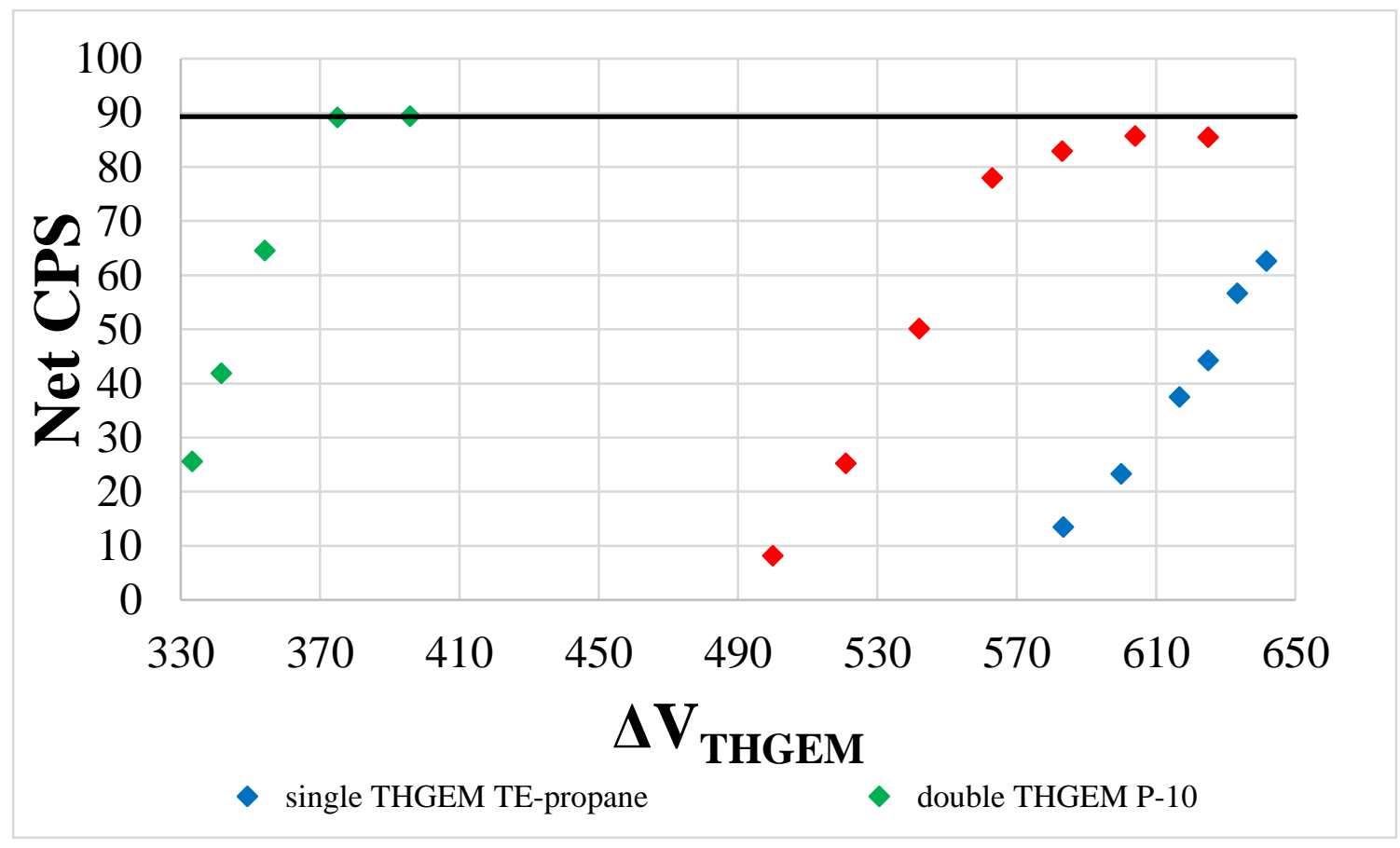

Figure III.10: Counting curves for 100 torr TE-propane and P10 gas using ${ }^{14} \mathrm{C}$. 


\section{5 ${ }^{14} \mathrm{C}$ Energy Calibration and MCNP6 Pulse Height Spectra Comparison}

In addition, compared with MCNP6 f8 tally results, experimental results were confirmed by the similar peak location and spectrum shape as the simulations (see Figure III.11). To calibrate the $\mathrm{x}$-axis of experimental data to match that of the simulation, channels were converted to energy utilizing pulser calibrations. ${ }^{244} \mathrm{Cm}$ and ${ }^{14} \mathrm{C}$ spectra measured at a fixed $\Delta \mathrm{V}_{\text {THGEM }}$ of $563 \mathrm{~V}$ were used in this example. For this fixed detector gain (i.e. same $\mathrm{HV}_{\text {cathode }}$ and $\mathrm{HV}_{\text {THGEM }}$ settings), energy calibration was completed using the corresponding peak channel for ${ }^{244} \mathrm{Cm}$, the calculated ${ }^{244} \mathrm{Cm} \mathrm{E}_{\mathrm{dep}}$, and pulser calibration data from the ORTEC 109A preamplifier test circuit. In the case of different applied HV and multiplication gain, a correction factor using the effective gain curve fit equations should also be used to account for different detector gain settings.

$$
E_{\text {dep } 14 C}=E_{\text {dep } 244 C m} \times \frac{V_{\text {pulser } 14 C}}{V_{\text {pulser } 244 C m p k c h}}
$$




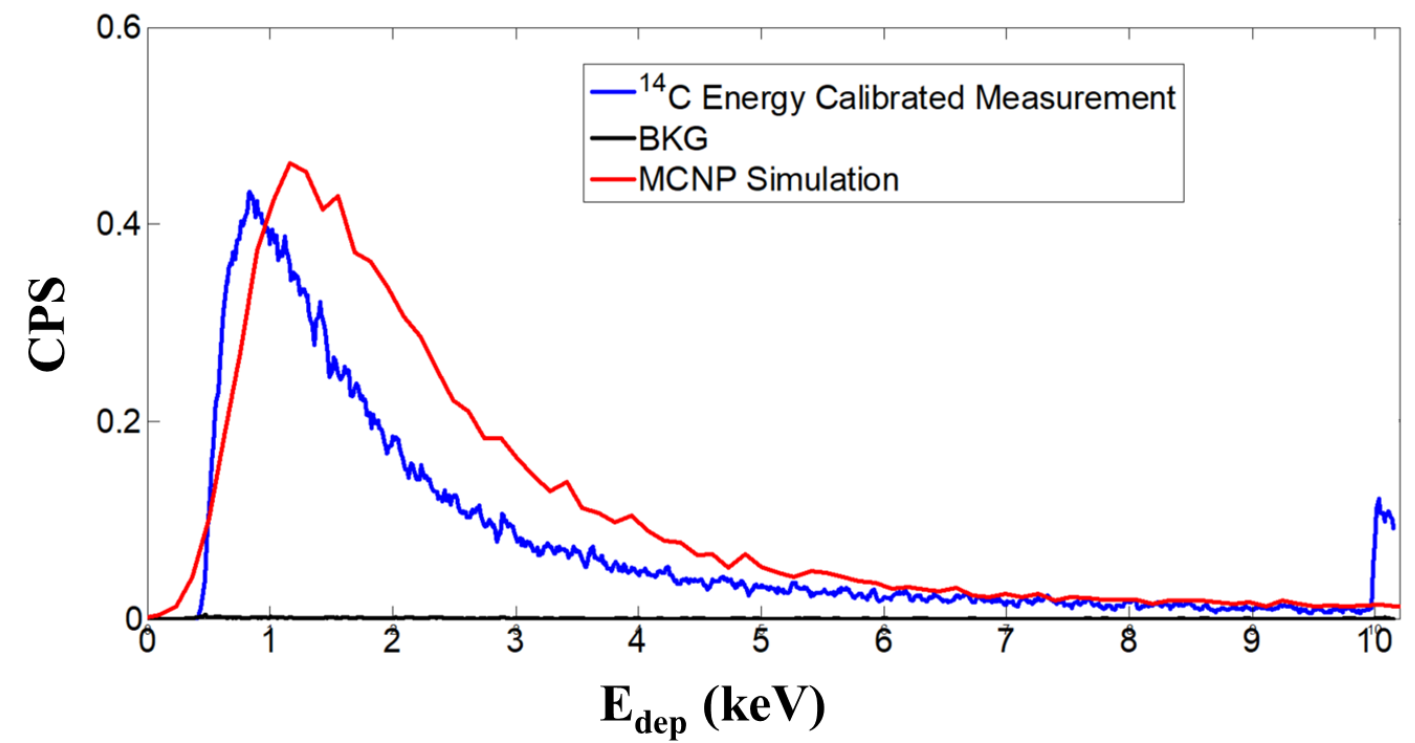

Figure III.11: Experimental data for double THGEM in 100 torr TE-propane at $\Delta \mathrm{V}_{\text {THGEM }}=563 \mathrm{~V}$ with MCNP6 simulated spectra for comparison. One noticeable feature is the rise in counts in the last few channels, attributed to particles that were binned in channels greater than 1024 .

To investigate the detector's operation at higher gas pressures, simulations were completed to observe the increase in $E_{\text {dep }}$ for increasing pressure. As gas pressure increases, a larger number of secondary electrons are created in the SV and the signal increases for a fixed energy beta particle. This results in a greater fraction of the total energy deposition spectrum being seen. Thus, for a fixed cut-off energy, a larger number of particles will be detected at higher pressures. It can be seen in Figure III.12 that not until 2280 torr (or $3 \mathrm{~atm}$ ) does the pulse height spectrum begin to approach the full $\mathrm{E}_{\mathrm{dep}}$ spectrum. Additionally, although a larger signal should be obtained at higher pressures due to more secondary electrons in the SV, higher operating voltages are also required which can limit the gain and increases probability of electrical discharge across components. 

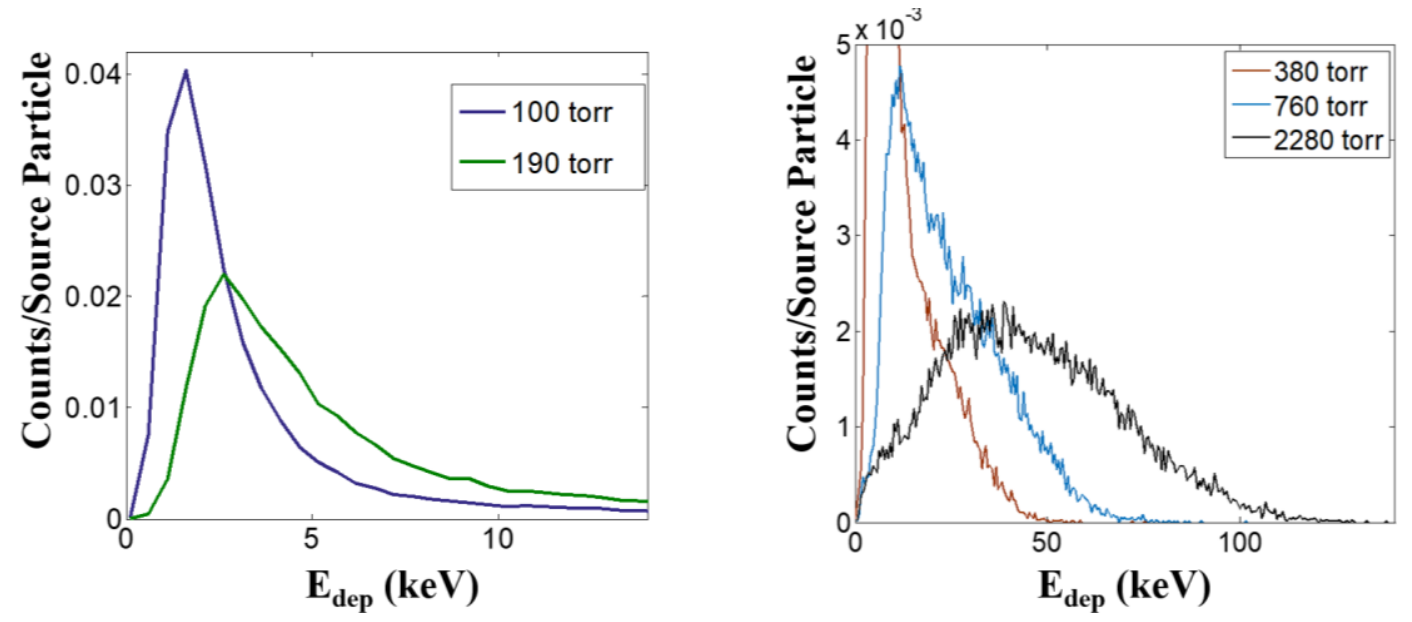

Figure III.12: MCNP6 simulation for ${ }^{14} \mathrm{C}$ in THGEM beta-ray detector at different gas pressures.

\section{6 ${ }^{14} \mathrm{C}$ Disk Source Beta-ray Stability Measurements}

Since it was not possible to ascertain the peak position of the beta-ray spectra accurately, gain stability was measured by recording the fluctuation in net count rate over time as the HV was left on. As displayed below in Figure III.13, for ${ }^{14} \mathrm{C}$ the detector displayed strong stability when left running for 4 hours. The count rate was also agreeable with the net count rate measured separately with the WPC. 


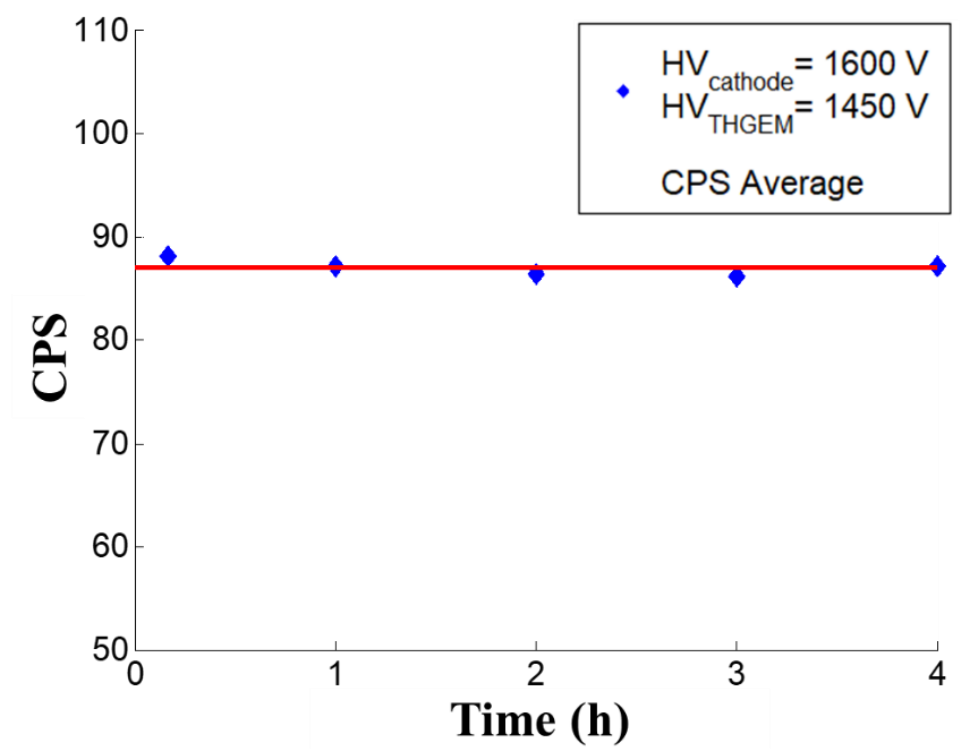

Figure III.13: ${ }^{14} \mathrm{C}$ source stability using net count rate $(\mathrm{cps})$ at $\mathrm{HV}_{\text {cathode }}=1600 \mathrm{HV}_{\text {THGEM }}=1450 \mathrm{~V}, 100$ torr TE-propane gas.

\section{7 ${ }^{90} \mathrm{Sr} / \mathrm{Y}$ Disk Source Beta-ray Measurements}

Subsequently, a higher activity $(48 \mathrm{kBq}){ }^{90} \mathrm{Sr} / \mathrm{Y}$ beta source was installed, which emits two beta-rays with $\beta_{\max }=0.546 \mathrm{MeV}$ and $\beta_{\max }=2.28 \mathrm{MeV}$. It was immediately evident that at higher net count rates $(>1000 \mathrm{cps})$, the overall measured count rate in Maestro was rapidly decreasing during measurements (see Figure III.14). This resulted in the failure to obtain a counting curve for this source due to the continuous decrease in count rate. However, the stability was still measured to determine how much the count rate would decrease by before stabilizing, which is shown to be approximately $375 \mathrm{cps}$ in Figure III.15. It can be seen that as gain decreased, count rate decreased by more than a factor of $3 . \mathrm{HV}_{\text {cathode }}$ was increased in an attempt to overcome the decrease in gain by 
increasing ion evacuation in the detector regions, however it did not sufficiently prevent the decrease.

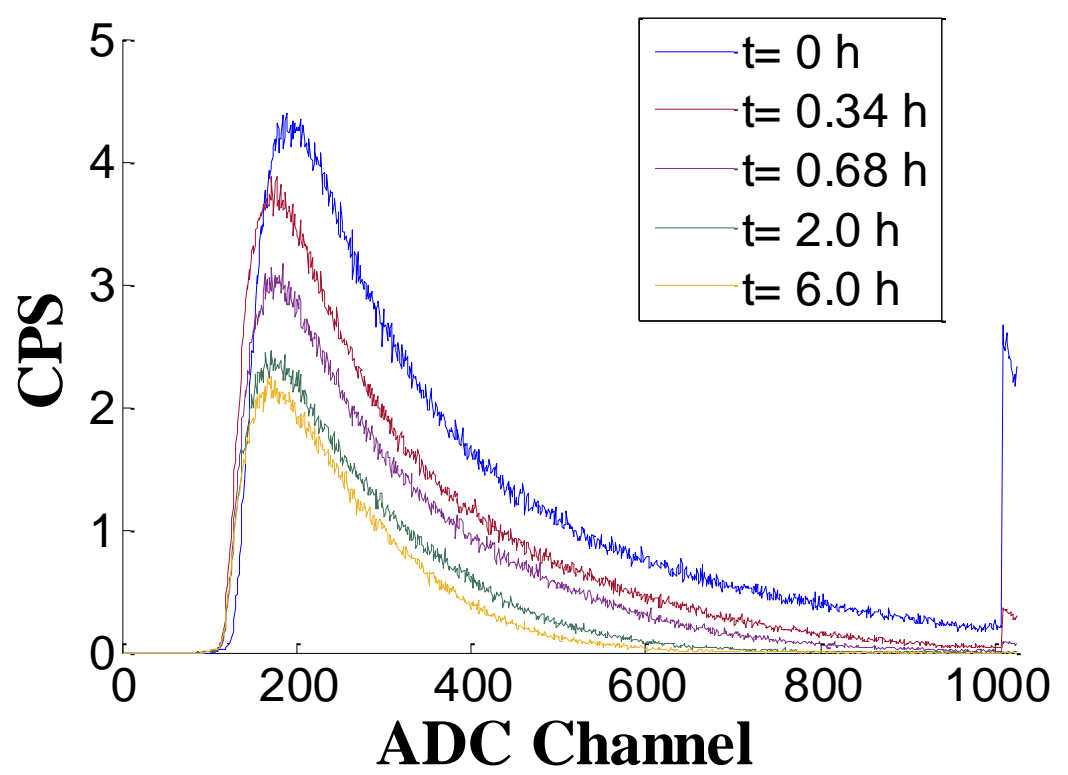

Figure III.14: Set of spectra used to measure stability for double-THGEM at $\mathrm{HV}_{\text {cathode }}=1500 \mathrm{~V}, \mathrm{HV}_{\mathrm{THGEM}}=$ $1350 \mathrm{~V}$ in 100 torr TE-propane gas.

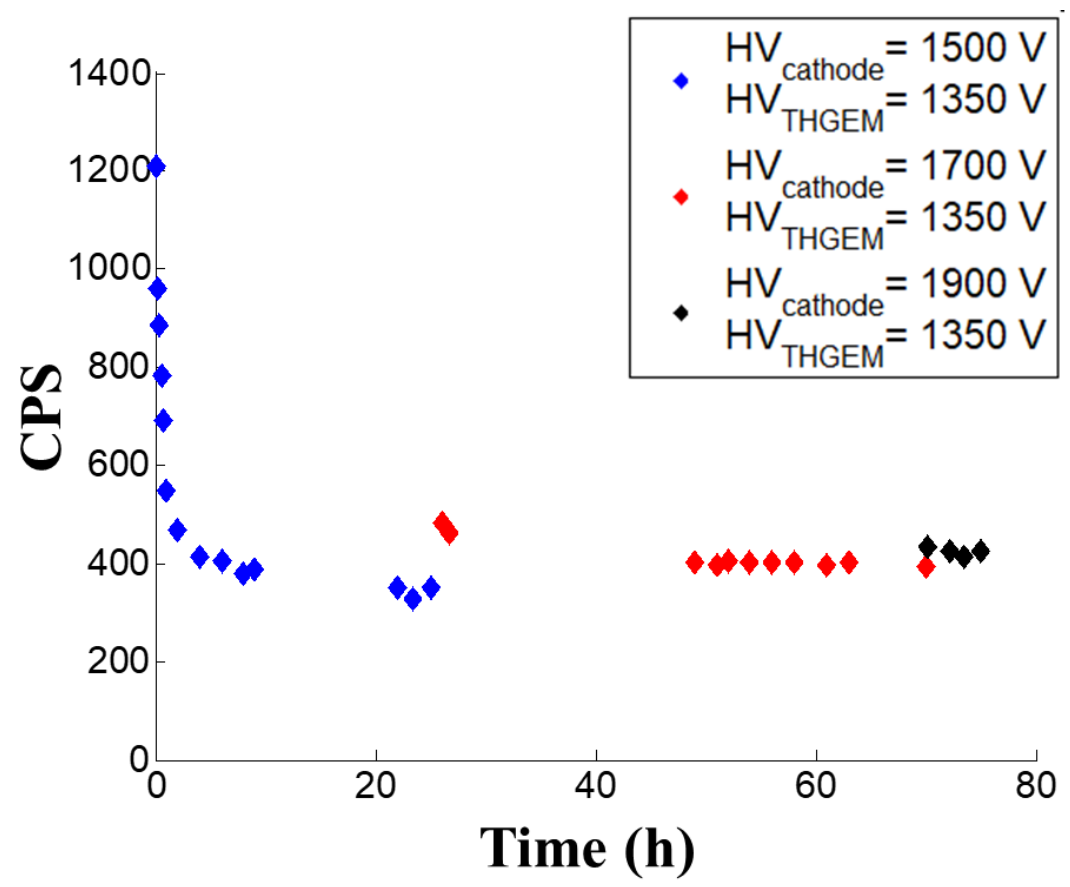

Figure III.15: ${ }^{90} \mathrm{Sr} / \mathrm{Y}$ source stability with $200 \mathrm{~V}$ increments for $\mathrm{HV}_{\text {cathode }}$ in 100 torr TE-propane. 


\section{Chapter IV.Discussion and Conclusions}

\subsection{Insulator Charge Build-up and Ion Back-flow (IBF) Effect on Gain}

The primary reason for a decrease in gain over time resulting in poor stability results is due to the space charge effect. The space charge effect occurs when positive ions are created by the incident beta-ray, and in the subsequent electron multiplication process. This modifies the E-fields throughout the detector, as heavy slower moving positive ions traverse towards the cathode resulting in Ion Back-flow (IBF). In addition, ions are deposited and build up positive space charge on the inner FR4 insulator surface of the THGEM holes, thus decreasing the E-field in the holes. A recent THGEM gain simulations study carried out by Correia et al. [34], calculated the gain evolution of rimless THGEMs in Ne and Ar based gas mixtures using a dedicated simulation toolkit integrated with Garfield++. It was reported that higher voltages require longer irradiation time or rate to reach stabilitization. Additionally, THGEM electrodes with thicker dimensions require less time to charge up and stabilize due to a greater increase in the spread of electrons during a townsend avalanche. This effect, in addition to IBF was also indicated in an experimental THGEM study using $\mathrm{H}_{2}$ and $\mathrm{D}_{2}$ gas by Cortesi et al. in 2015 [44]. Similar studies [45,46] using GEM detectors have confirmed the IBF effect on gain, and suggest that higher count rates result in a reduction of gain, as well as a more prominent effect for each additional GEM stage in triple configuration. It is described that the space charge effect alters the E-fields of transfer and multiplication regions, thereby reducing gain. Solutions to the problem involve evacuating ions faster, using less stages 
of multiplication, in addition to the potential use of graphene to allow movement of electrons but not ions through GEM layers.

\subsection{Conclusion}

An advanced THGEM based beta-ray detector has been fabricated and tested. With an active area of $42 \times 42 \mathrm{~mm}$, thickness of $0.4 \mathrm{~mm}$, and source-to-detector distance typically set to $10 \mathrm{~mm}$, the detector was successfully operated in single and doubleTHGEM configurations in low pressure TE-propane and P10 gas. Necessary components including an aluminum vacuum chamber, copper collimators, collection anodes, and voltage dividers were customized for the detector. After optimizing the drift E-field to $150 \mathrm{~V} / \mathrm{cm}$ using a ${ }^{244} \mathrm{Cm}$ alpha source with a $\mathrm{d}=1 \mathrm{~mm}$ and $\mathrm{h}=2 \mathrm{~mm}$ collimator, gain stability and effective gain measurements were carried out. Alpha spectra were fit using an EMG function to monitor the peak position. MCNP6 simulations were used to verify the alpha peak shape, including high energy tail. Reliable long term gain stability was observed using the alpha peak channel over several days. High detector gains above $10^{4}$ were measured using P10 gas in a double-THGEM configuration, with $4.5 \mathrm{~mm}$ and $3 \mathrm{~mm}$ spacing between the transfer region and collection gap respectively before the preamp was saturated. Using a second collimator with a diameter of $23 \mathrm{~mm}$, beta-ray source measurements were carried out using ${ }^{14} \mathrm{C}$ and ${ }^{90} \mathrm{Sr} / \mathrm{Y}$. The count rate plateau was reached for ${ }^{14} \mathrm{C}$ in $\mathrm{P} 10$ gas using the double-THGEM configuration at $\Delta \mathrm{V}_{\mathrm{THGEM}} \approx 375 \mathrm{~V}$. The maximum achievable count rate was in strong agreement with WPC measurements. 
MCNP6 simulations were used to verify experimentally measured beta-ray spectra by accurately modelling the source and detector. Counting curves for the relatively much higher activity ${ }^{90} \mathrm{Sr} / \mathrm{Y}$ beta-ray source were not successful due to decrease in gain over time. The decrease in gain manifesting as a rapid decrease in count rate was measured. Investigations are underway to resolve this effect.

\subsection{Future Work}

Despite the detector performing well when measuring ${ }^{14} \mathrm{C}$ at a low count rate, performance can still be improved by reducing the positive space charge effects, primarily by preventing ion backflow. Methods to reduce this are still under investigation, and improvement is imperative to ensure adequately stable operation at high count rates. One simple approach could be to operate with a single-THGEM, as this would allow positive ions to travel to the cathode much more efficiently which would cause less extreme distortion in the E-fields. In addition, a very recently designed Multi-Mesh THGEM (MM-THGEM) detector by Olivera and Cortesi [47] has shown great capability in the reduction of IBF several times lower compared to standard double-THGEM detectors. This is accomplished by implanting two electrode meshes, which function as multipliers with uniform electric fields inside the holes, and also aid in trapping a large quantity of positive ions migrating to the drift region. Two meshes were installed by gluing and sealing stainless steel mesh between three thin PCBs, which includes two PCBs with single copper layers for the top and bottom of the MM-THGEM and one 
middle insulator PCB without copper clad for separating the meshes. Other methods include using gas-mixtures which allow greater ion mobility, only a single multiplication stage, or graphene layers. Graphene is one of thinnest materials existing, and was proven to reduce IBF as it allows high transparency for electrons but is impermeable for ions when suspended above the holes [46]. A more detailed investigation into the effect count rate has on gain stability is required, which could be accomplished by reducing the count rate of the ${ }^{90} \mathrm{Sr} / \mathrm{Y}$ source using a filter and observing the result. With less particles depositing energy, it is expected there will be a decrease in positive ions degrading the Efields and reducing gain.

Other structural modifications include increasing the surface area by using an array of THGEMs and readout boards. This would likely be one of the next areas of investigation, as hexagonal THGEMs are already fabricated each with a $24.9 \mathrm{~cm}^{2}$ active area, and can easily interface with each other to greatly increase the active area (see Figure IV.1). A new structure would need to be designed to support the THGEMs, readout boards, and cathode within the chamber. In addition, by changing the solid copper cathode structure to a wire mesh, larger area monitoring capabilities could be created. A window designed for the chamber could enable it to function as a portable contamination meter, which would warrant investigation into window material type, thickness, and fill gas pressure. 


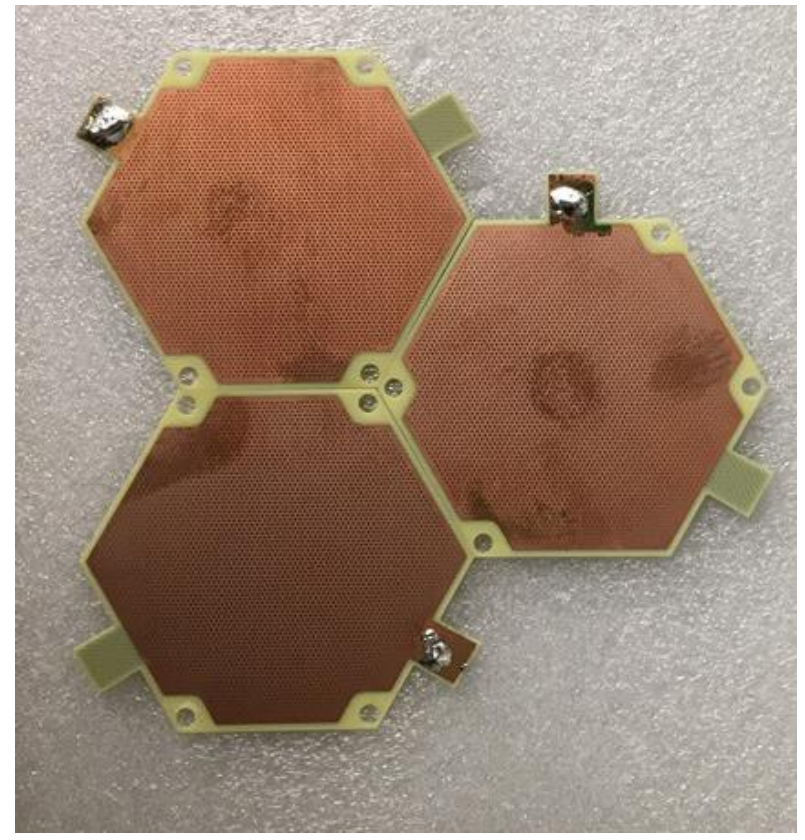

Figure IV.1: Three hexagonal THGEMs used to increase surface area.

Another area of interest would involve comparing the THGEM based beta-ray detectors response to commercially available detectors (windowed and windowless) in terms of efficiency and minimum detectable activity (MDA). This would involve comparisons with commercial fixed and portable instruments using accessible specification sheets, and benchmarking using direct measurements from instruments readily available on campus or ones that can be economically purchased.

In terms of computations, a more extensive Monte Carlo simulation study could be performed to better optimize the source and detector geometry. This includes modifying the SV dimensions and fill gas. In addition, E-field and avalanche simulations could be conducted to better understand the consequence different fill gas types have on gain and the IBF effect generated from positive ions. Finally, a better statistical treatment 
of the data, including the calculation of coefficient of variation (to measure the relative data variability to average peak channel) from gain stability measurements. It is also recommended to use centroid channel instead of peak channel from an EMG fit as a better indicator of change in gain.

Lastly, it would be ideal to test the function of a THGEM detector for in-air tritium sampling. Using a controlled environment and gas flow equipment, the effect of introducing the tritiated air and counting gas (such as P10) into the chamber can be experimentally determined. As tritiated air is introduced it is suspected gain will decrease due to high electron attachment of $\mathrm{O}_{2}$. The ideal percentage of sampled air and counting gas will need to be investigated. 


\section{Appendix A.1: MCNP6 Deck for ${ }^{244} \mathrm{Cm}$ Alpha Source with Collimator}

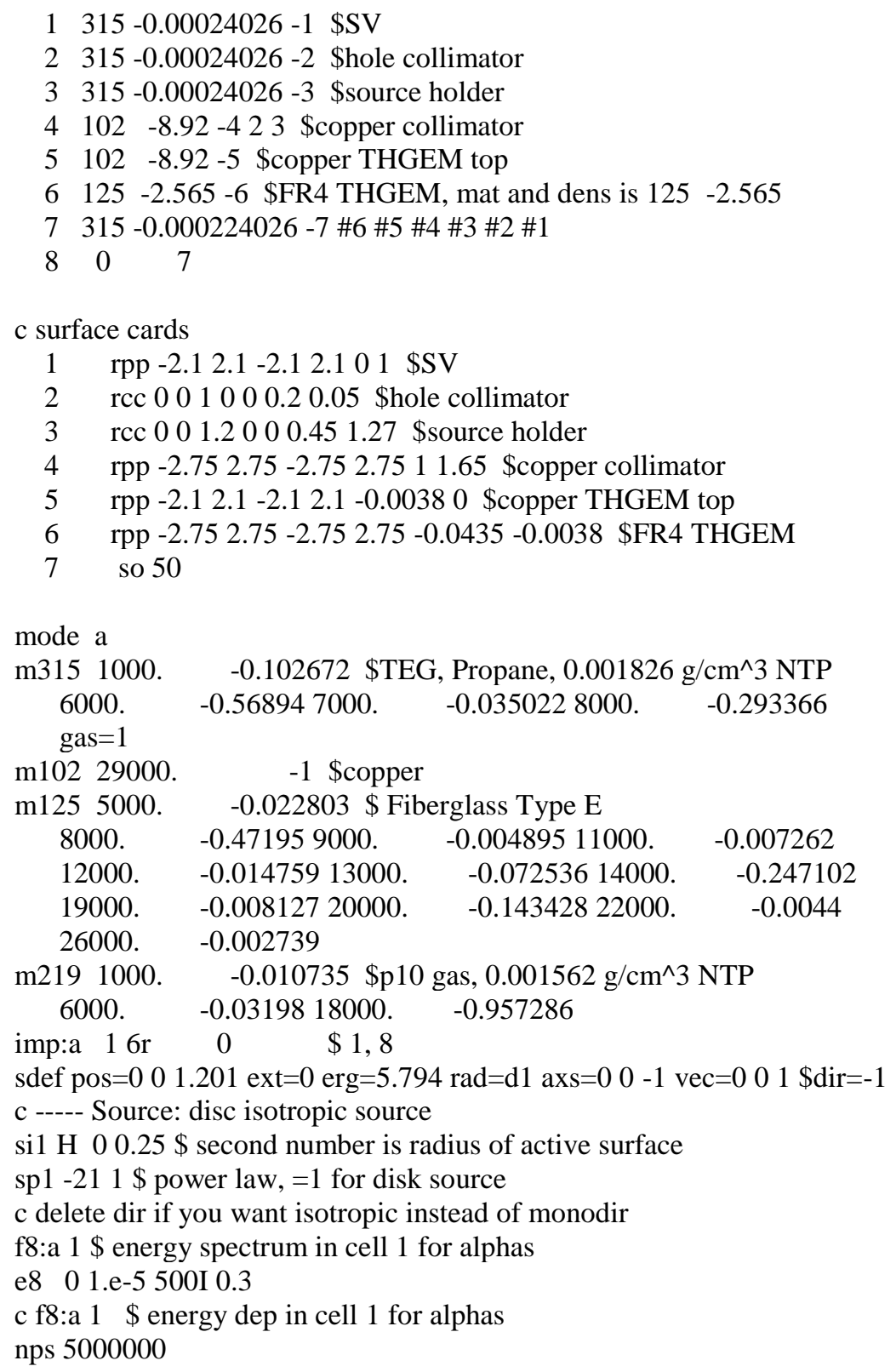




\section{References}

[1] S.H. Byun, G.M. Spirou, A. Hanu, W. V. Prestwich, A.J. Waker, Simulation and first test of a microdosimetric detector based on a thick gas electron multiplier, IEEE Trans. Nucl. Sci. 56 (2009) 1108-1113. doi:10.1109/TNS.2008.2009214.

[2] E. Rutherford, H. Geiger, An Electrical Method of Counting the Number of Alpha Particles from Radio-Active Substances, Proc. R. Soc. A Math. Phys. Eng. Sci. 81 (1908) 141-161. doi:10.1098/rspa.1908.0065.

[3] M.T. Ryan, J.W. Poston, A half century of health physics : 50th anniversary of the Health Physics Society, Lippincott Williams \& Wilkins, 2005.

https://books.google.ca/books/about/A_Half_Century_of_Health_Physics.html?id=qCebx PjdSBUC\&redir_esc=y (accessed November 28, 2017).

[4] J.A. Simpson, A Precision Alpha-Proportional Counter, Rev. Sci. Instrum. 18 (1947) 884893. doi:10.1063/1.1740874.

[5] First Generation Gas Flow Alpha Proportional Counter, (2011). https://www.orau.org/ptp/collection/proportional counters/simpson.htm (accessed January 1, 2018).

[6] G. Charpak, R. Bouclier, T. Bressani, J. Favier, Č. Zupančič, The use of multiwire proportional counters to select and localize charged particles, Nucl. Instruments Methods. 62 (1968) 262-268. doi:10.1016/0029-554X(68)90371-6.

[7] H. Klingenmeyer, Invention and development of the multiwire proportional chamber, in: Semin. Nobel Prize. Part. Phys., University of Heidelberg, 2007. https://www.physi.uniheidelberg.de/ reygers/seminars/2015/nobel_prizes_in_particle_physics/talks/klingenmey er_mwpc.pdf (accessed January 2, 2018).

[8] V. Peskov, Advances in Micro-Pattern Gaseous Detectors and their Applications, (n.d.). https://arxiv.org/ftp/arxiv/papers/0906/0906.5215.pdf (accessed January 3, 2018).

[9] F. Sauli, GEM: A new concept for electron amplification in gas detectors, Nucl. Instruments Methods Phys. Res. Sect. A Accel. Spectrometers, Detect. Assoc. Equip. 386 (1997) 531-534. doi:10.1016/S0168-9002(96)01172-2.

[10] F. Sauli, The gas electron multiplier (GEM): Operating principles and applications, Nucl. Instruments Methods Phys. Res. Sect. A Accel. Spectrometers, Detect. Assoc. Equip. 805 (2016) 2-24. doi:10.1016/J.NIMA.2015.07.060.

[11] R.A. Surette, J. Dubeau, Tritium discrimination using cluster size information from a DGEM detector, Nucl. Instruments Methods Phys. Res. Sect. A Accel. Spectrometers, Detect. Assoc. Equip. 539 (2005) 433-440. doi:10.1016/j.nima.2004.09.039.

[12] A. Hanu, Development of a THGEM Imaging Detector with Delay Line Readout, Open Access Diss. Theses. (2014). http://digitalcommons.mcmaster.ca/opendissertations/8587.

[13] S. Darvish, Development of a 2d thgem microdosimetric detector, (2016). https://macsphere.mcmaster.ca/handle/11375/20445 (accessed June 21, 2018). 
[14] Z. Anjomani, A.. Hanu, W.V. Prestwich, S.H. Byun, Development of a multi-element microdosimetric detector based on a thick gas electron multiplier, Nucl. Instruments Methods Phys. Res. Sect. A Accel. Spectrometers, Detect. Assoc. Equip. 847 (2017) $117-$ 124. doi:10.1016/J.NIMA.2016.11.051.

[15] D. Vartsky, I. Israelashvili, M. Cortesi, L. Arazi, A.E. Coimbra, L. Moleri, E. Erdal, D. Bar, M. Rappaport, S. Shchemelinin, E.N. Caspi, O. Aviv, A. Breskin, Liquid-Xe detector for contraband detection, Nucl. Instruments Methods Phys. Res. Sect. A Accel.

Spectrometers, Detect. Assoc. Equip. 824 (2016) 240-242.

doi:10.1016/j.nima.2015.10.104.

[16] C.M.B. Monteiro, L.M.P. Fernandes, J.F.C.A. Veloso, C.A.B. Oliveira, J.M.F. dos Santos, Secondary scintillation yield from GEM and THGEM gaseous electron multipliers for direct dark matter search, Phys. Lett. B. 714 (2012) 18-23.

doi:10.1016/J.PHYSLETB.2012.06.066.

[17] J.B.R. Battat, I.G. Irastorza, A. Aleksandrov, T. Asada, E. Baracchini, J. Billard, G. Bosson, O. Bourrion, J. Bouvier, A. Buonaura, K. Burdge, S. Cebrin, P. Colas, L. Consiglio, T. Dafni, N. D’Ambrosio, C. Deaconu, G. De Lellis, T. Descombes, A. Di Crescenzo, N. Di Marco, G. Druitt, R. Eggleston, E. Ferrer-Ribas, T. Fusayasu, J. Galïn, G. Galati, J.A. Garcïa, J.G. Garza, V. Gentile, M. Garcia-Sciveres, Y. Giomataris, N. Guerrero, O. Guillaudin, A.M. Guler, J. Harton, T. Hashimoto, M.T. Hedges, F.J. Iguaz, T. Ikeda, I. Jaegle, J.A. Kadyk, T. Katsuragawa, S. Komura, H. Kubo, K. Kuge, J. Lamblin, A. Lauria, E.R. Lee, P. Lewis, M. Leyton, D. Loomba, J.P. Lopez, G. Luzïn, F. Mayet, H. Mirallas, K. Miuchi, T. Mizumoto, Y. Mizumura, P. Monacelli, J. Monroe, M.C. Montesi, T. Naka, K. Nakamura, H. Nishimura, A. Ochi, T. Papevangelou, J.D. Parker, N.S. Phan, F. Pupilli, J.P. Richer, Q. Riffard, G. Rosa, D. Santos, T. Sawano, H. Sekiya, I.S. Seong, D.P. Snowden-Ifft, N.J.C. Spooner, A. Sugiyama, R. Taishaku, A. Takada, A. Takeda, M. Tanaka, T. Tanimori, T.N. Thorpe, V. Tioukov, H. Tomita, A. Umemoto, S.E. Vahsen, Y. Yamaguchi, M. Yoshimoto, E. Zayas, Readout technologies for directional WIMP Dark Matter detection, Phys. Rep. 662 (2016) 1-46. doi:10.1016/j.physrep.2016.10.001.

[18] Argos-AB Family of Gas Flow Whole Body Contamination Monitors, (n.d.). http://www.canberra.com/products/hp_radioprotection/pdf/Argos-AB-Family-SSC40575.pdf (accessed January 2, 2018).

[19] J. Burnham, Chapter 8- Internal Dose, in: Radiat. Prot. Green B., PLGS, Toronto, 2001: pp. 236-248. https://canteach.candu.org/Content Library/20031308.pdf.

[20] CNSC, Tritium Releases and Dose Consequences in Canada in 2006 Part of the Tritium Studies Project INFO-0793, 2009.

[21] M.M. Bé, V.P. Chechev, 14C-Comments on evaluation of decay data, (2012). http://www.nucleide.org/DDEP_WG/Nuclides/C-14_com.pdf (accessed January 5, 2018).

[22] T.-D. Le, Management of Carbon-14 Emissions and Environmental Impacts at Ontario Power Generations Nuclear, in: RETS/REMP Work., 2011. http://www.clearwater.org/wpcontent/uploads/2017/03/Exhibit-10-14-Emissions-CANDU-copy.pdf (accessed January 6, 2018).

[23] W. Sohn, D.-W. Kang, W.-S. Kim, An Estimate of Carbon-14 Inventory at Wolsong 
Nuclear Power Plant in the Republic of Korea, J. Nucl. Sci. Technol. J. J. Nucl. Sci. Technol. J. Nucl. Sci. Technol. 408 (2003) 22-3131.

doi:10.1080/18811248.2003.9715397.

[24] Application Note: iPCM12 Installed Personnel Contamination Monitor, (2016) 3. https://www.thermofisher.com/order/catalog/product/AE0229A?SID=srch-srp-AE0229A (accessed January 6, 2018).

[25] Ludlum Model 44-92 Beta Gamma Detector, (2014). http://ludlums.com/images/data_sheets/M44-92.pdf (accessed January 6, 2018).

[26] A. Kamal, Chapter 1- Passage of Charged Particles Through Matter, Berlin Heidelberg, 2014. doi:10.1007/978-3-642-38655-8.

[27] R. Nave, Threshold for Relativistic Effects, (n.d.). http://hyperphysics.phyastr.gsu.edu/hbase/Relativ/rellim.html (accessed January 8, 2018).

[28] D. Wagenaar, CHARGED PARTICLE INTERACTIONS, JPNM Phys. (1994). http://www.med.harvard.edu/JPNM/physics/didactics/physics/charged/lect.html (accessed January 8, 2018).

[29] Radiation Physics Lecture 4 Interactions of Charged Particles with Matter, (n.d.). http://www.physics.usyd.edu.au/ kuncic/lectures/RP4_slides.pdf (accessed April 4, 2018).

[30] W. Prestwich, Chapter 6- Charged Particle Interactions, (n.d.). https://www.science.mcmaster.ca/radgrad/images/775CourseResources/775_ch6.pdf (accessed January 7, 2018).

[31] NIST estar, (n.d.). https://physics.nist.gov/PhysRefData/Star/Text/ESTAR.html (accessed April 3, 2018).

[32] G.E. Knoll, Radiation Detection and Measurement, Third, John Wiley \& Sons, Inc, Ann Arbor, Michigan, 2000.

https://phyusdb.files.wordpress.com/2013/03/radiationdetectionandmeasurementbyknoll.p df (accessed April 19, 2018).

[33] S.H. Byun, G.M. Spirou, A. Hanu, W. V. Prestwich, A.J. Waker, Simulation and First Test of a Microdosimetric Detector Based on a Thick Gas Electron Multiplier, IEEE Trans. Nucl. Sci. 56 (2009) 1108-1113. doi:10.1109/TNS.2008.2009214.

[34] P.M.M. Correia, M. Pitt, C.D.R. Azevedo, A. Breskin, S. Bressler, C.A.B. Oliveira, A.L.M. Silva, R. Veenhof, J.F.C.A. Veloso, Simulation of gain stability of THGEM gasavalanche particle detectors, (2017). https://arxiv.org/pdf/1711.02073.pdf (accessed April 2, 2018).

[35] T. Francke, V. Peskov, Innovative applications and developments of micro-pattern gaseous detectors, Information Science Reference, 2014. https://books.google.ca/books?id=MRGXBQAAQBAJ\&dq=raether+limit\&source=gbs_na vlinks_s (accessed May 13, 2018).

[36] Z. Anjomani, MULTIPLIER-BASED MULTI-ELEMENT MICRODOSIMETRIC DETECTOR, (2017) 1-42. 
[37] I. Krajcar-Bronić, D. Srdoc, A comparison of calculated and measured W values in tissueequivalent gas mixtures., Radiat. Res. 137 (1994) 18-24.

http://www.ncbi.nlm.nih.gov/pubmed/8265783 (accessed May 21, 2018).

[38] H. Tawara, N. Ishida, J. Kikuchi, T. Doke, Measurements of the W values in argon, nitrogen, and methane for 0.93 to $5.3 \mathrm{MeV}$ alpha particles, Nucl. Instruments Methods Phys. Res. Sect. B Beam Interact. with Mater. Atoms. 29 (1987) 447-455.

doi:10.1016/0168-583X(87)90055-3.

[39] NIST astar stopping-power and range tables for helium ions, (n.d.). https://physics.nist.gov/PhysRefData/Star/Text/ASTAR.html (accessed May 22, 2018).

[40] R. Dinner, Electron Attachment in Gaseous Detectors, MIT, 2000. http://cyclo.mit.edu/theses/rdinnermain.pdf.

[41] P. Povinec, A study of proportional counter optimization for long-term counting, Nucl. Instruments Methods. 163 (1979) 363-368. doi:10.1016/0029-554X(79)90120-4.

[42] G.M. Orchard, A.J. Waker, Transit time of electrons and gas gain effects in P-10 and Ar+CO 2, Nucl. Instruments Methods Phys. Res. Sect. A Accel. Spectrometers, Detect. Assoc. Equip. 764 (2014) 236-240. doi:10.1016/j.nima.2014.07.057.

[43] A. Sharma, F. Sauli, First Townsend coefficient measured in argon based mixtures at high fields, Nucl. Instruments Methods Phys. Res. A . (1993) 420-424. https://ac.elscdn.com/016890029390802O/1-s2.0-016890029390802O-main.pdf?_tid=5eed6635-628c42ad-9769-66af8134efca\&acdnat=1527096046_c0fd806ac0e2d182f695b27352849027 (accessed May 23, 2018).

[44] M. Cortesi, J. Yurkon, W. Mittig, D. Bazin, S. Beceiro-Novo, A. Stolz, Studies of THGEM-based detector at low-pressure Hydrogen/Deuterium, for AT-TPC applications, (2015). doi:10.1088/1748-0221/10/09/P09020.

[45] R. Chechik, A. Breskin, C. Shalem, M. Cortesi, G. Guedes, V. Dangendorf, D. Vartsky, D. Bar, Progress in Thick GEM-like. (THGEM)-based Detectors, (2006). https://indico.cern.ch/event/25658/contributions/575264/attachments/450360/624470/snic _chechik.pdf (accessed April 2, 2018).

[46] P. Thuiner, Ion space-charge effects in multi-GEM detectors: challenges and possible solutions for future applications, (2016). http://cds.cern.ch/record/2238855? ln=en (accessed April 2, 2018).

[47] R. De Olivera, M. Cortesi, First performance evaluation of a Multi-layer Thick Gaseous Electron Multiplier with in-built electrode meshes -MM-THGEM, (2018). https://arxiv.org/ftp/arxiv/papers/1804/1804.04643.pdf (accessed June 20, 2018). 